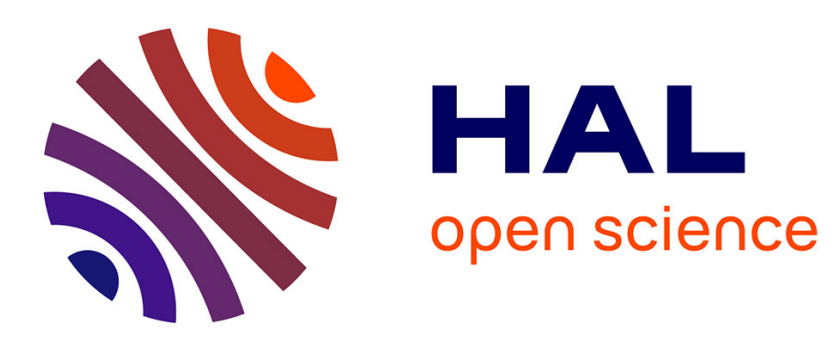

\title{
Probing Ion Channel Structure and Function Using Light-Sensitive Amino Acids
}

Viktoria Klippenstein, Laetitia Mony, Pierre Paoletti

\section{To cite this version:}

Viktoria Klippenstein, Laetitia Mony, Pierre Paoletti. Probing Ion Channel Structure and Function Using Light-Sensitive Amino Acids. Trends in Biochemical Sciences, 2018, 10.1016/j.tibs.2018.02.012 . hal-01781105

\section{HAL Id: hal-01781105 \\ https://hal.science/hal-01781105}

Submitted on 29 Apr 2018

HAL is a multi-disciplinary open access archive for the deposit and dissemination of scientific research documents, whether they are published or not. The documents may come from teaching and research institutions in France or abroad, or from public or private research centers.
L'archive ouverte pluridisciplinaire HAL, est destinée au dépôt et à la diffusion de documents scientifiques de niveau recherche, publiés ou non, émanant des établissements d'enseignement et de recherche français ou étrangers, des laboratoires publics ou privés. 


\section{Trends in Biochemical Sciences}

\section{PROBING ION CHANNEL STRUCTURE AND FUNCTION USING LIGHT-SENSITIVE AMINO ACIDS \\ --Manuscript Draft--}

\begin{tabular}{|l|l|}
\hline Manuscript Number: & TIBS-D-18-00034R1 \\
\hline Article Type: & Review \\
\hline Corresponding Author: & Pierre Paoletti \\
\hline Paris, FRANCE \\
\hline First Author: & Viktoria Klippenstein \\
\hline Order of Authors: & Viktoria Klippenstein \\
\hline Laetitia Mony \\
\hline Pbstract: & \begin{tabular}{l} 
Pierre Paoletti \\
\hline Approaches to remotely control and monitor ion channel operation with light are fast \\
expanding in the field of biophysics and neuroscience. A recent development to directly \\
introduce light-sensitivity into proteins relies on the genetic code expansion technique \\
by utilizing photo-sensitive unnatural amino acids (UAAs). Introduction of UAAs results \\
in unique molecular level control, and combined with the maximal spatio-temporal \\
resolution and poor invasiveness of light, enables direct manipulation and interrogation \\
of ion channel functionality. Here, we review the diverse applications of light-sensitive \\
UAAs in two superfamilies of ion channels - voltage- and ligand-gated ion channels - \\
and summarize the existing UAA tools, their mode of action, potential, caveats, and \\
technical considerations to illuminate ion channel structure and function.
\end{tabular} \\
\hline
\end{tabular}




\title{
PROBING ION CHANNEL STRUCTURE AND FUNCTION USING LIGHT-SENSITIVE AMINO ACIDS
}

\author{
Viktoria Klippenstein*, Laetitia Mony* and Pierre Paoletti
}

Institut de Biologie de l'ENS (IBENS)

CNRS UMR8197, INSERM U1024

Ecole Normale Supérieure

Université PSL

46 rue d'Ulm, 75005 Paris, France

*: equal contribution

Correspondence to: Pierre Paoletti (pierre.paoletti@ens.fr)

\section{Keywords:}

optopharmacology; fluorescence; optogenetics; ion channels; unnatural amino acids (UAAs); photo-sensitive tethered ligands (PTLs)

\section{Acknowledgements:}

Research of authors is supported by the French government under the program 'Investissements d'Avenir' (ANR-10-LABX-54 MEMO LIFE and ANR-11-IDEX-0001-02 PSL* Research University), the European Research Council (ERC Advanced Grant \#693021 to PP), the European Commission (Marie Sklodowska-Curie fellowship H2020-MSCA-IF-2015 Grant \#701467 to LM), and the Deutsche Forschungsgemeinschaft (DFG; postdoctoral fellowship \#KL 2935/1-1 to VK). 


\section{ABSTRACT}

Approaches to remotely control and monitor ion channel operation with light are fast expanding in the field of biophysics and neuroscience. A recent development to directly introduce light-sensitivity into proteins relies on the genetic code expansion technique by utilizing photo-sensitive unnatural amino acids (UAAs). Introduction of UAAs results in unique molecular level control, and combined with the maximal spatio-temporal resolution and poor invasiveness of light, enables direct manipulation and interrogation of ion channel functionality. Here, we review the diverse applications of light-sensitive UAAs in two superfamilies of ion channels - voltage- and ligand-gated ion channels - and summarize the existing UAA tools, their mode of action, potential, caveats, and technical considerations to illuminate ion channel structure and function. 


\section{ION CHANNELS AS MEDIATORS OF ELECTRICAL SIGNALING}

All cells in the body - from epithelial, secretory, cardiac, neuronal, to muscle cells - contain ion channels. Amongst others, their activity mediates muscular contraction, vascular tone, electrical signaling in the heart as well as hormone secretion and cell proliferation. Ion channels also allow accurate brain function by controlling neuronal excitability and synaptic transmission [1]. Being large protein complexes embedded into biological membranes, ion channels do not only specify the functional properties of cells, but also of specific cellular sites and compartments. Electrical signaling across the membrane is shaped by controlled ion flow through their conducting pore, which is a remarkably precise and rapid biological event.

Ion channels are highly heterogeneous proteins on a structural and functional level. This heterogeneity is mostly mediated by their multimeric nature due to different combinations of subunits, creating a diversity of ion channel subtypes. Distinct subtypes can differ in their mode of activation, their conductive properties, and their pharmacological behavior. For example, $\mathrm{GABA}_{A}$ receptors, a class of pentameric ion channels, can incorporate 16 different subunits, giving rise to at least 20 distinct subtypes in the brain with different biophysical and pharmacological properties [2]. Similarly, $>10$ subtypes of NMDA receptors (NMDARs), ionotropic glutamate receptors (iGluRs) that cluster at excitatory synapses, co-exist in the central nervous system [3]. This is even far outreached by potassium channels, whose subunits are encoded by no less than 77 genes, thus representing the most diverse ion channel class [4].

Two ion channel superfamilies mediate a plethora of time-defined cellular processes: voltage-gated ion channels (VGICs) and ligand-gated ion channels (LGICs). VGICs are represented by a multiplicity of ion channel types, among others four-domain voltage-gated sodium and calcium channels, and one- or multi-domain channels such as voltage-gated potassium channels and channels from the transient receptor potential (TRP) family. Typically, all of those open their pore in dependence of changes in the membrane voltage, which is mediated by a voltage-sensing domain (VSD) [5, 6]. On the other hand, LGICs are activated by ligands that bind extra-cellular domains. They can be trimeric (such as P2X receptors), tetrameric (all iGluRs including AMPA, NMDA, and kainate receptors), or pentameric (e.g. $\mathrm{GABA}_{\mathrm{A}}$ and nicotinic acetylcholine receptors [nAChRs]) [7]. In addition to their gating core (i.e. the ion pore and the signal-sensing domains), most classes of ion channels contain accessory domains or are associated with auxiliary subunits, further diversifying their functional and signaling properties $[8,9]$. 
LGICs and VGICs are furthermore major potential drug targets, involved in a wide variety and regulation processes of these channels in native conditions, where their subunit composition and microenvironment can influence their function, is key in drug design. So far, electrophysiology, molecular biology, biochemistry, pharmacology and structural biology (Xray crystallography and cryo-electron microscopy) have brought a wealth of information on these questions. However, these methods missed either structural, spatial, or temporal resolution, limiting our understanding of ion channel structure and function. Thus, methods with high spatial resolution and fast enough to synchronize with the channels' dynamic mode of action during normal and disease states are required.

Light confers this required high spatio-temporal resolution. Various techniques such as fluorescence spectroscopy, 'optopharmacology' [11] and 'optogenetic pharmacology' (also coined 'synthetic optogenetics' [12]) exploit of the power of light, by engineering lightresponsiveness into ion channels and receptors (Table 1). Here, we review recent advances to directly introduce light-sensitivity into ion channels using genetically-encoded, photoreceptive unnatural amino acids (UAAs). We discuss a group of light-sensitive UAAs, carrying different photo-chemical properties, and provide an overview of their implementation to address key questions regarding ion channel structure, gating, regulation, assembly, and physiology. By embedding the genetic UAA methodology into the large and fast growing field of molecular optogenetics, we highlight its strong potential, but also its limitations, and future perspectives in biomolecular engineering and cellular physiology.

\section{ENDOWING LIGHT-SENSITIVITY TO ION CHANNELS}

Traditional optogenetics has routinely utilized naturally occurring light-sensitive ion channels such as channelrhodopsins to study cellular activity by light. To address biological questions of endogenous, natively expressed ion channels, however, supplementary tools are required. For decades, conventional site-directed mutagenesis, pharmacological tools, or genetic manipulations (e.g. knock-out animals) have been the major techniques to provide direct functional information about ion channels. These have obvious limitations such as deficient molecular and cellular specificity, insufficient drug delivery, poor reversibility, or physiological compensatory effects. Above all, the development of subtype-specific pharmacological agents remains challenging, calling for novel approaches that allow precise molecular control over specific ion channel isoforms.

In close collaboration between biologists and chemists, various tools have been evolved to directly engineer light-responsiveness into ion channels, which are naturally non-receptive 
to optical stimulation. Hereby, a distinction is drawn between the use of light-activatable on iGluRs [13]; optopharmacology), and post-translational labeling approaches of genetically modified target ion channels (optogenetic pharmacology and fluorescence monitoring) [11, 14-16] (Table 1). In the latter category, the powerful combination of optics, genetics, and pharmacology results in high molecular level control, which, combined with the unique properties of light, enables direct manipulation and interrogation of ion channel functionality. This allows to remotely investigate ion channel molecular operation and structural mechanisms, and provides means to photo-control their mode of action at the physiological level.

Currently, the leading strategy makes use of cysteine-mutated receptors labeled by tethered fluorophores or photo-sensitive tethered ligands (PTLs; Table 1). PTLs can comprise cysteine-tethered, photo-reactive compounds such as the benzophenone photocrosslinker BPMTS [17, 18] (Table 1). Most PTLs, however, are based on a photoisomerizable azobenzene moiety (see Glossary) that undergoes reversible toggling between two states upon exposure to light - a compact cis and a stretched trans configuration [19, 20]. PTLs also contain a cysteine-reactive group, usually a maleimide, and a pharmacologically active head group, which can turn on, turn off, or modulate channel activity (e.g. MAG [21]; Table 1). Since labeling is performed post-translationally, PTL functional loci are restricted to solvent accessible, extra-cellular protein sites [20]. Moreover, the resulting photo-control is limited at a certain maximum, never reaching completeness [22]. Although reducing agents may be applied to increase cysteine accessibility [11], the existence of mixed ion channel populations including labeled, light-sensitive, and unlabeled versions is inevitable. Finally, the turnover cycle of ion channels at cellular membranes requires cysteine re-labeling, when using PTLs [11]. PTL conjugation has been broadly applied in neuronal VGICs and LGICs to efficiently photo-control their activity [11, 12, 16, 20], and crucially, it has pioneered native ion channel photo-modulation in vivo [22-24].

Recently, an attractive strategy to engineer light-responsiveness into ion channels hit the field of molecular optogenetics and became a powerful alternative to chemical labeling. It relies on the site-specific introduction of light-sensitive UAAs into ion channels by genetic code expansion to achieve optical control of their activity (Table 1). Below, we discuss the strong and fast expanding potential of these light-sensitive UAAs to study ion channels with excellent molecular and spatio-temporal precision, with a focus on important methodological aspects and the biological questions addressed so far. 


\section{INCORPORATING UAAS INTO ION CHANNELS}

The chemical composition of UAAs is broad including fluorescent, light-sensitive, redoxactive or bioconjugatable side-chains [25, 26]. Their distinctive feature is the ab initio introduction into the polypeptide sequence, just at the assigned time of its biosynthesis, thus not requiring any post-translational modifications. Combined with their minimal size of only one side-chain, UAAs are endowed with a theoretical 'absolute' site tolerance [27]. This aspect is particularly important for ion channels, whose pore domain, embedded in the membrane bilayer, is largely solvent-inaccessible. Most channels further contain key regulatory intra-cellular domains that cannot be labeled post-translationally using the cysteine chemistry. The UAA methodology hijacks the translation machinery through the reassignment of a stop codon, which is, at present, the primary technique to site-specifically introduce UAAs. Traditionally, TAG 'amber' stop codons are used to be suppressed by a specific tRNA (the suppressor tRNA), aminoacylated with a UAA of interest [26, 28-30]. Sufficient amounts of the UAA within the cell are achieved by supplementing the compound into the cellular growth medium or directly injecting it into the cell.

UAA aminoacylation of tRNAs has first been carried out in vitro, followed by direct microinjection into Xenopus laevis oocytes, where the UAA is straight delivered onto the amber codon of a protein coding sequence $[28,31]$. This way of protein manipulation requires the synthesis of the UAA followed by chemical tRNA aminoacylation in a test tube, offering a high-rate screening of UAAs and a high level of flexibility [28,30]. Being however restricted to the application in large Xenopus oocytes, this chemical approach limits structural and functional ion channel studies that require more native cellular systems. Also, stoichiometric consumption and lack of in cellulo regeneration of the UAA-decorated tRNA, following cellular injection, limits the amount of the synthesized protein.

From the emerging demand to introduce UAAs into proteins expressed in mammalian cells, a more recent approach evolved, which relies on the genetic encoding of UAAs [26, 29, 32] (Figure 1). This technique utilizes bio-orthogonal tRNA/synthetase pairs, specifically engineered for the introduction of a particular amino acid. All components required for 'nonsense' stop codon suppression, including the amber version of the desired protein and the specific tRNA/synthetase pair, are introduced into the cell in the form of plasmids. Once processed within the cell, the tRNA is charged with the UAA by its cognate synthetase to suppress the amber codon on the protein mRNA. Bio-orthogonality and cellular compatibility of tRNA/synthetase pairs must accompany each other [26, 29, 32]. Thus, these pairs undergo longsome directed evolutionary processes in the lab, which can correspond to several years of work. Consequently, the genetic approach is overall slower and offers less flexibility compared to chemical UAA introduction. However, once evolved, this technique 
greatly extends the possibilities of UAA utilization to a variety of systems, such as mammalian cell lines and neuronal cultures [33-35], brain slices [33, 36, 37], and even whole organisms including C. elegans [38], Drosophila [39], Zebrafish [40], and mice [33, 40, 41]. Beneficially, constant regeneration of the aminoacylated tRNA enables its repeated use within the cell (Figure 1) and thus high-scale protein yields. Compared to post-translational labeling with PTLs, usage of UAAs shares commonalities and differences that need to be carefully studied before deciding on the use on one or the other technique (Table 2). Furthermore, to overcome obstacles and facilitate the establishment of the UAA methodology in the lab, as well as to ensure its smooth implementation and an appropriate interpretation of the obtained data, a few major points are summarized in Box $\mathbf{1}$.

The following sections summarize recent advances in using light-sensitive and fluorescent UAAs to control and monitor diverse ion channel functionalities. Detailed technical and historical aspects of both the chemical and genetic UAA approach have been recently reviewed in detail elsewhere [29-31, 42].

\section{TRACKING ION CHANNEL STRUCTURAL DYNAMICS USING FLUORESCENT UAAS (fUAAs)}

The capacity of fluorescent probes to undergo FRET or sense their direct chemical environment has been extensively used to monitor conformational changes of ion channels in real time. Moreover, when combined to electrical measurements as performed using VCF, it provides a unique correlation between structural rearrangements and gating steps (Figure 2A) [43]. Recently, fUAAs have proven to be powerful probes for FRET and VCF studies.

In a pioneering study, using nonsense suppression with in vitro aminoacylated tRNAs, Cohen et al. [44] introduced an alanine derivative of 6-dimethylamino-2-acyl-naphtalene (Aladan, Figure 2B) into buried sites of Shaker and Kir2.1 potassium channels. The Xenopus oocyte translation machinery is in fact capable of incorporating fluorophores as bulky as BODIPYFL [45], Cy3, and Cy5 into ion channels [46] (Figure 2B), suggesting that a wide variety of fUAAs can be potentially introduced into proteins using in vitro aminoacylated tRNAs. A few bio-orthogonal tRNA/synthetase pairs have also been developed for genetic encoding of fUAAs [25], such as small, naphthalene-derived fUAAs like (7-hydroxycoumarin4-yl)ethylglycine [47] and Dansyl-Alanine [48] (Figure 2C). (7-hydroxycoumarin-4$\mathrm{yl}$ )ethylglycine was incorporated at the pore entrance of NaK channels as a probe of ion selectivity [49]; Dansyl-Alanine, on the other hand, in the VSD of CiVSP, a voltagedependent enzyme containing a VSD similar to VGICs [50]. Interestingly, by introducing the 
fluorophore at the external or internal extremity of the voltage-sensing helix S4, changes in cell membrane potential could be optically monitored.

A major leap forward in the field came with the recent development of 3-(6acetylnaphtalen-2-ylamino)-2-aminopropanoic acid (Anap, Figure 2C) [51, 52]. In regard of brightness and quantum yield. Anap is comparable to dansyl or coumarin fluorophores, however it displays strongly enhanced environmental sensitivity [52]. Anap is also a good FRET donor to GFP [53] or to transition metal ions [54-56], thus representing a powerful probe of protein conformational changes. It was first introduced in the VSD of Shaker [57], whose gating mechanisms have been thoroughly studied by VCF (reviewed in ref [58]). By introducing either Anap or a cysteine, labeled with tetramethylrhodamine maleimide (TMR), at the same S4 helix site, Anap appeared equally potent as TMR to track the voltage-sensing steps, but also reported additional conformational changes occurring at highly hyperpolarized potentials. Simultaneous motion tracking at both extremities of the critical S4 helix was even achieved by orthogonally inserting the two fluorophores at sites opposite to the membrane, Anap close to S4 intra-cellular end and TMR at the extra-cellular end (Figure 2D), something rather challenging using post-translational labeling only. The observed asynchronous fluorescence changes at the two S4 ends suggest different intra- and extra-cellular conformational rearrangements during channel transition to the open state. Since then, the application of Anap to study ion channel structural rearrangements has expanded rapidly. In further VCF studies, Anap was incorporated in the intra-cellular catalytic domain of CiVSP to monitor its voltage-dependent rearrangements [59], as well as in the extra-cellular and transmembrane domains of glycine receptors to study the mechanisms of partial agonism [60]. The versatility of UAA site insertion, in particular intra-cellularly, was further exploited to monitor the structural rearrangements during gating of key intra-cellular regulatory domains of cyclic nucleotide-gated (CNG) [54], TRPV1 [56] and EAG-like (ELK) voltage-gated potassium channels [55].

In summary, the main advantage of genetic fUAA incorporation compared to posttranslational labeling lies in their unrivaled site tolerance. Furthermore, due to their compact structures, fUAAs like Anap are more tightly associated to the protein than tethered fluorophores. Therefore, they allow more accurate distance measurements in FRET experiments and are likely to sense subtle conformational changes that would be averaged out by organic tethered fluorophores, which are often connected to the protein via a flexible linker [57]. However, the small size of fUAAs is also a disadvantage. They are usually excited by UV-range wavelengths (360 $\mathrm{nm}$ for Anap in water [52]) that induce strong autofluorescence from the cell cytoplasm. Moreover, they have an extinction coefficient $(\varepsilon)$ and quantum yield (Q) lower than most common organic fluorophores $\left(\varepsilon=19500 \mathrm{~cm}^{-1} \mathrm{M}^{-1}\right.$, 
$Q=0.22$ in aqueous buffer for Anap [56] vs $\varepsilon=93000 \mathrm{~cm}^{-1} \mathrm{M}^{-1} ; \mathrm{Q}=0.79$ in PBS for

AlexaFluor® 456). Considering that proteins incorporating fUAAs are usually expressed at lower levels than their wild-type or cysteine-mutated counterparts, the use of fUAAs may lead to a lower fluorescence signal-to-noise ratio. Examples in the literature show, nevertheless, that it is possible to obtain similar levels of fluorescence changes with Anap or tethered fluorophores (Figure 2D, also compare refs [60] and [61]), likely because the strong environment sensitivity of this fUAA compensates for its poorer spectral properties. Anap can also yield fluorescence under two-photon excitation [62], raising the potential to study dynamics of ion channels in intact tissues.

\section{MANIPULATING ION CHANNEL FUNCTION USING LIGHT-SENSITIVE UAAS}

Decorating ion channels with light-responsive UAAs affords an artificial control mechanism that is orthogonal to the natural one provided by evolution (change in membrane potential, ligand binding). A variety of light-sensitive UAAs, carrying photo-crosslinking, photoswitchable, photo-cleavable or photo-caged side-chains, have been successfully used to obtain remote and highly precise control of ion channel function.

Photo-chemical UAA decaging was initially performed in potassium channels and nAChRs expressed in Xenopus oocytes, using various protection groups attached to cysteine, glycine, or tyrosine [28, 63-65]. In a typical experiment, channel opening is hindered by the presence of the photo-caged UAA, carrying a photo-labile group (cage), which is removed upon illumination with wavelength-specific light. Although the recovered electrophysiological responses following photolysis were overall small, this initial work has provided first fundamental information about the kinetics of decaging. Recently, a photoactivatable, inwardly rectifying potassium channel (PIRK), carrying the first genetically encoded photo-caged UAA, was designed [36]. The UAA 4,5-dimethoxy-2-nitrobenzylcysteine (Cmn; Figure 3A,B), a photo-caged cysteine, was introduced into the pore of Kir2.1. When placed directly within the ion channel pore, the bulky UAA occluded it, keeping the channel in a non-conductive state (Figure 3A). Following expression in rat hippocampal neurons, brief UV pulses restored the outward potassium current, as detected by the suppression of neuronal firing. In this work, the authors also demonstrated the first expression and optical modulation of a light-sensitive UAA-containing ion channel in vivo. The UAA expression machinery was introduced by means of in utero electroporation and electrophysiological recordings were performed from acute embryonic mouse neocortex slices. The development of PIRK demonstrates a big achievement in the UAA world, opening new vistas in studying ion channel function in vivo. Since then, a photo-caged lysine was 
successfully incorporated into a GFP-amber model protein in the mouse brain of live mice [33].

Another form of photo-chemical uncaging was further performed for photo-cleavable UAAs, which generate a 'caged' protein backbone that can be cut in two upon UV illumination [28]. Photolysis of the UAA 2-(nitrophenyl)glycine (Npg) was utilized to induce peptide backbone cleavage in a loop of $\mathrm{GABA}_{\mathrm{A}}$ receptors, resulting in an alteration of their pharmacology [66]. Generally, the ability to modify the protein main chain using UAAs is a valuable tool for mechanistic investigations of ion channels [67]. The approach remains limited to cells amenable to direct UAA-tRNA injection however, since evolved tRNA/synthetase pairs for genetic encoding of the corresponding UAAs are still lacking.

To date, the most commonly used light-sensitive UAAs in the ion channel field are photocrosslinking probes. In particular, the two photo-crosslinkers Azido-phenylalanine (AzF) and Benzoyl-phenylalanine (BzF or Bpa) have been widely utilized for their potential to map protein-protein and protein-ligand interactions as well as their functional consequences [68]. Both UAAs are photo-chemically activated by light at the UV range, which generates radicals that covalently crosslink a C-H bond-containing chemical group in close vicinity (Figure 3CF) [69]. AzF and BzF can both be efficiently incorporated into proteins using specifically evolved AzF and BzF tRNA/synthetase pairs [70] that allow achieving robust levels of expression of various LGICs and VGICs in Xenopus oocytes and mammalian cells (as follows).

The power of BzF photo-crosslinking has been exploited to investigate if the VGIC KCNQ1 directly interacts with the $\beta$-subunit KCNE1 [71]. Following BzF introduction into the auxiliary subunit, rapid and complete silencing of channel activity upon UV illumination was observed, indicative of covalent crosslinking to KCNQ1 (Figure 3C). Furthermore, BzF photo-crosslinking helped to clarify a variable stoichiometry model for the channel complex composition, a previously contentious issue. Taking advantage of light-induced crosslinking events in real time, subsequent work further investigated the dynamics of this interaction during the gating process, revealing a state-dependent interaction between KCNQ1 and KCNE1 [72]. These studies demonstrate the strong potency of genetically encoded photocrosslinkers to probe not only protein interactions directly in their cellular context, but also their dynamics during channel gating.

The powerful combination of light with the precise molecular control using photocrosslinking UAAs has further allowed capturing dynamic functional states underlying operation of iGluRs, ion channel receptors that mediate excitatory synaptic transmission in the brain. During their activation cycle, iGluRs pass through different short-lived conductance 
states [73]. In particular, studying the ultrafast gating properties of AMPARs with activation AMPA and NMDA receptors, positions that undergo pronounced conformational rearrangements during receptor gating. Following expression in either Xenopus oocytes or mammalian cell lines, photo-crosslinking was induced by UV stimulation and detected by simultaneous electrophysiological recordings. In AMPARs, by placing BzF between two adjacent agonist-binding domains (ABDs), applications of UV pulses promoted irreversible and potent inactivation of glutamate-activated responses in a state-dependent manner [75] (Figure 3E,F). Depending on the BzF position within the ABDs, receptor silencing was observed in either active, desensitized, or resting states, thus providing important information about inter-subunit rearrangements during gating transitions. In NMDARs, AzF photocrosslinking has been primarily used to study the N-terminal domains (NTDs), which lie the most distal to the transmembrane domain and display strong allosteric capacity [76]. By placing AzF at strategic locations at a NTD upper lobe dimer interface, both UV-induced potentiation and inhibition of channel activity were detected [35, 77], demonstrating the potential of this interface to mediate bi-directional control of NMDAR activity. AzF photocrosslinking of NTDs was performed in oocytes [77, 78], HEK cells, and primary hippocampal neurons [35], with consistent light-dependent effects. The design of light-sensitive AMPA and NMDA receptors has established a basis for using UAA photo-crosslinkers to study structurefunction mechanisms in large receptor complexes such as iGluRs. Recent work showing AzF incorporation into the GFP-amber model protein in generated transgenic Zebrafish and mouse lines further demonstrates the strong potential to use photo-crosslinking UAAs in vivo [40].

The irreversible nature of photo-crosslinking allows accumulation of ion channels or receptors trapped in the same state, resulting in strong biophysical phenotypes. Thus, physically trapped complexes can be isolated and confirmed biochemically [71, 75]. To obtain functional reversibility, as abundantly described when using PTLs, azobenzene-based photo-switchable UAAs (PSAAs) were developed [79]. PSAAs were recently implemented to generate a family of NMDARs that can be accurately and reversibly controlled by light (Figure 3G-I) [80]. PSAA introduction within different subunits and receptor domains enabled rapid, stable, and reproducible photo-control, achieved by light-dependent toggling between the trans and cis states of the azobenzene side-chain (Figure $3 \mathrm{H}$ ). PSAA toggling within the receptor $\mathrm{ABDs}$ allowed control of co-agonist sensitivity, while targeting transmembrane pore sites enabled optical modulation of gating and permeation properties. Moreover, both subunit-specific and bi-directional (photo-inactivation and photo-potentiation) receptor control 
were achieved [80]. PSAAs combine the powerful properties of UAAs and azobenzenes to reversibly manipulate ion channel function. Particularly, they can be incorporated at solventinaccessible sites, thus providing new means to probe and directly control transmembrane domain motions with atomic scale definition. Some PSAAs contain additional functional groups (e.g. benzyl chloride) that allow formation of covalent bridges with adjacent cysteines in a light-dependent manner [79]. Future applications of photo-controllable bridge formations in ion channels that can combine reversibility (provided by the azobenzene moiety) and irreversibility (provided by cysteine-reactive groups) will allow more precise optical control, and potentially increase the mechanical forces exerted by the UAA to directly control channel gating. Enhanced biocompatibility is also to be expected with new PSAA versions harboring red-shifted azobenzene moieties [81].

Taken together, light-sensitive UAAs offer great qualities as optopharmacological tools to study ion channel structural and functional properties at the molecular level, and crucially, in real time. When placed at key moving sites or interfaces, they provide valuable tools to address key questions regarding ion channel structure, gating, regulation, or assembly. Depending on the UAA of interest, channel activity can be either irreversibly turned on or turned off (e.g. photo-caged or photo-crosslinking UAAs), or be manipulated in a reversible manner (photo-switchable versions). So far, structure-function investigations and protein interaction studies have been primarily performed in oocytes or mammalian cell lines. However, first reports support the feasibility of UAA application in native preparations, opening new possibilities to study biological ion channel processes in a complex and preserved environment.

\section{IN VIVO USAGE OF UAAS: POTENTIAL AND CHALLENGES}

Optical manipulation of proteins such as ion channels in vivo is worthy of pursuit for obvious reasons. The accompanied gain of molecular control obtained by generating light-sensitive versions of entire ion channel families would greatly contribute to a deeper understanding of their physiological roles during normal and disease states, information highly valuable in drug design. With its site flexibility and genetic encodability, the UAA methodology provides highly precise and selective tools to dissect channel functionality at the sub-cellular, cellular, tissue, and model organism level. The existence of various UAAs carrying specific chemical groups offers a rich functional spectrum for light-mediated ion channel intervention, which can occur on a time scale compatible with their biological activity. Furthermore, incorporation of two different UAAs into the same ion channel (e.g. to unite distinct chemical properties) or even combination of different optopharmacological tools is conceivable [82]. 
Being multi-component tools, optogenetic pharmacology approaches are still technically vivo, essential requirements include (Table 2): (I) introduction and co-expression of genes encoding the mutant target and the tRNA/synthetase pair; (ii) administration of the UAA; and (iii) efficient delivery of light [11]. Although expression of the genetic components in mammalian cell lines or primary neuronal cultures following transient transfection has been proven efficient enough to obtain sufficient levels of mutant ion channels [34, 35], absolute expression yields are usually lower compared to their wild-type counterparts [34, 80]. Thus, delivery of the UAA expression machinery into multi-cellular systems poses challenges. Viral vectors have been used to include UAAs into proteins expressed in neural stem cells, primary neuronal cultures, organotypic brain slices, and even living mice [33, 37, 50]. In utero electroporation is an alternative [36]. Crucially, to ensure an adequate suppression of the amber codon, driven by efficient expression of a functional suppressor tRNA, multiple tRNA copies are required $[33,36,41,50]$. In order to obtain maximal protein expression levels, the number of viral vectors should be kept low, preferably carrying several genes separated by IRES sequences [36, 50]. Favorably, by viral delivery, a high number of cells are infected, which can ideally deliver many copies of the tRNA/synthetase pair. High cell-type specificity, and thus very local modifications in signaling, can be obtained by characteristic vector design. Less beneficial, the over-expressed subunits co-exist with endogenous wild-type subunits, resulting in a mixture of ion channel populations. This can be avoided by the design of knock-in mice, where light-sensitive subunit versions substitute their wild-type counterparts to obtain 'clean' ion channel populations [22, 83]. Finally, the genetic code can be expanded and inherited following generation of transgenic mouse lines that directly incorporate the specific tRNA/synthetase pair into their genome [40, 41]. First, zebrafish and mouse strains allowing incorporation of the photocrosslinker AzF have become available [40]. Custommade transgenic mouse strains are, in theory, conceivable for any tRNA/synthetase pairs and should demonstrate a strong potential to study native ion channel function.

UAAs can be directly injected into the brains of living mice $[33,36]$, either solitary or in the format of dipeptides to increase delivery [36]. Alternatively, they can be supplied through drinking water or food with no described toxic side-effects [33, 40]. Depending on the tissue of interest, intraperitoneal injections can also be considered [41]. However, sufficient bioavailability of the UAA at the target tissue and cells may be an issue during long-lasting in vivo experiments.

To obtain maximal optical effects, an optimal light supply should be assured. Light should efficiently penetrate the tissue of interest without inducing damage, preferably by the use of longer wavelengths. Here, the obtained knowledge from utilizing PTLs is highly 
valuable [22]. If it is inevitable to use short wavelengths in the UV range, light pulses should of light can fine-tune channel modulation [36]. As for PTLs [84, 85], red-shifted UAA versions with enhanced light penetration and tissue tolerance will be of prime importance in vivo [81]. With respect to specificity, a number of potential limitations including 'leakage' through unspecific amber codon suppression [78, 86] or suppression of amber stop codons within other genes, are to be considered, but remain to be fully evaluated (Table 2).

In summary, although using UAAs is currently mostly performed in heterologous expression systems, these promising foregoing studies demonstrate that photo-modulating ion channel function in vivo is, despite obvious obstacles, within reach. Once resolved, genetic encodability, spatiotemporal accuracy, and molecular specificity should greatly contribute to our understanding of native LGICs and VGICs.

\section{CONCLUDING REMARKS AND FUTURE PERSPECTIVES}

UAAs can be efficiently introduced into ion channels to render them light-sensitive. To date, these ion channels can be robustly expressed in diverse cellular systems, tissues, and living animals. The nanoscale photo-control can either act as a biophysical probe, or a remote actuator of ion channel activity, thus providing novel insights into their gating properties, pharmacology, and interacting partners with excellent spatio-temporal precision. The broad applicability of light-sensitive UAAs in molecular engineering and cellular physiology emphasizes its significance in the fields of optogenetic pharmacology and optical monitoring of protein conformational changes. A wealth of structural and functional information can be gained using this technology in vitro on recombinant proteins, while, in vivo, it has the potential to address fundamental questions regarding native ion channel or receptor biology (see Outstanding Questions for examples). Next generation opto-chemical UAA tools will expand their functional repertoire and generate further light-sensitive ion channel families, whose activity will be fine-tuned without interfering with their natural activation. Given its broad utility and functionally rich implementation, UAA insertion is poised to become increasingly popular for investigating protein structure and function. 


\section{REFERENCES}

1. Hille, B. (2001) Ionic Channels of Excitable Membranes, Sinauer.

2. Mohler, H. (2006) GABA(A) receptor diversity and pharmacology. Cell Tissue Res 326 (2), 505-16.

3. Paoletti, P. et al. (2013) NMDA receptor subunit diversity: impact on receptor properties, synaptic plasticity and disease. Nat Rev Neurosci 14 (6), 383-400.

4. Coetzee, W.A. et al. (1999) Molecular diversity of K+ channels. Ann N Y Acad Sci 868, 233-85.

5. Catterall, W.A. (2017) Forty Years of Sodium Channels: Structure, Function, Pharmacology, and Epilepsy. Neurochem Res 42 (9), 2495-2504.

6. Yu, F.H. and Catterall, W.A. (2004) The VGL-chanome: a protein superfamily specialized for electrical signaling and ionic homeostasis. Sci STKE 2004 (253), re15.

7. Lemoine, D. et al. (2012) Ligand-gated ion channels: new insights into neurological disorders and ligand recognition. Chem Rev 112 (12), 6285-318.

8. Dolphin, A.C. (2016) Voltage-gated calcium channels and their auxiliary subunits: physiology and pathophysiology and pharmacology. J Physiol 594 (19), 5369-90.

9. Greger, I.H. et al. (2017) Structural and Functional Architecture of AMPA-Type Glutamate Receptors and Their Auxiliary Proteins. Neuron 94 (4), 713-730.

10. Imbrici, P. et al. (2016) Therapeutic Approaches to Genetic Ion Channelopathies and Perspectives in Drug Discovery. Front Pharmacol 7, 121.

11. Kramer, R.H. et al. (2013) Optogenetic pharmacology for control of native neuronal signaling proteins. Nat Neurosci 16 (7), 816-23.

12. Berlin, S. and Isacoff, E.Y. (2017) Synapses in the spotlight with synthetic optogenetics. EMBO Rep 18 (5), 677-692.

13. Volgraf, M. et al. (2007) Reversibly caged glutamate: a photochromic agonist of ionotropic glutamate receptors. J Am Chem Soc 129 (2), 260-1.

14. Fehrentz, T. et al. (2011) Optochemical genetics. Angew Chem Int Ed Engl 50 (51), 12156-82.

15. Gautier, A. et al. (2014) How to control proteins with light in living systems. Nat Chem Biol 10 (7), 533-41.

16. Kienzler, M.A. and Isacoff, E.Y. (2017) Precise modulation of neuronal activity with synthetic photoswitchable ligands. Curr Opin Neurobiol 45, 202-209.

17. Ding, S. and Horn, R. (2001) Slow photo-cross-linking kinetics of benzophenone-labeled voltage sensors of ion channels. Biochemistry 40 (35), 10707-16.

18. Horn, R. et al. (2000) Immobilizing the moving parts of voltage-gated ion channels. J Gen Physiol 116 (3), 461-76. 
19. Beharry, A.A. and Woolley, G.A. (2011) Azobenzene photoswitches for biomolecules. Chem Soc Rev 40 (8), 4422-37.

20. Broichhagen, J. and Trauner, D. (2014) The in vivo chemistry of photoswitched tethered ligands. Curr Opin Chem Biol 21, 121-7.

21. Volgraf, M. et al. (2006) Allosteric control of an ionotropic glutamate receptor with an optical switch. Nat Chem Biol 2 (1), 47-52.

22. Lin, W.C. et al. (2015) A Comprehensive Optogenetic Pharmacology Toolkit for In Vivo Control of GABA(A) Receptors and Synaptic Inhibition. Neuron 88 (5), 879-891.

23. Levitz, J. et al. (2016) A Toolkit for Orthogonal and in vivo Optical Manipulation of Ionotropic Glutamate Receptors. Front Mol Neurosci 9, 2.

24. Berry, M.H. et al. (2017) Restoration of patterned vision with an engineered photoactivatable G protein-coupled receptor. Nat Commun 8 (1), 1862.

25. Xiao, H. and Schultz, P.G. (2016) At the Interface of Chemical and Biological Synthesis: An Expanded Genetic Code. Cold Spring Harb Perspect Biol 8 (9).

26. Liu, C.C. and Schultz, P.G. (2010) Adding new chemistries to the genetic code. Annu Rev Biochem 79, 413-44.

27. Wang, L. and Schultz, P.G. (2004) Expanding the genetic code. Angew Chem Int Ed Engl 44 (1), 34-66.

28. Beene, D.L. et al. (2003) Unnatural amino acid mutagenesis in mapping ion channel function. Curr Opin Neurobiol 13 (3), 264-70.

29. Chin, J.W. (2017) Expanding and reprogramming the genetic code. Nature 550 (7674), 53-60.

30. Leisle, L. et al. (2015) Incorporation of Non-Canonical Amino Acids. Adv Exp Med Biol $869,119-51$.

31. Pless, S.A. and Ahern, C.A. (2013) Unnatural amino acids as probes of ligand-receptor interactions and their conformational consequences. Annu Rev Pharmacol Toxicol 53, 21129.

32. Wang, L. et al. (2006) Expanding the genetic code. Annu Rev Biophys Biomol Struct 35, 225-49.

33. Ernst, R.J. et al. (2016) Genetic code expansion in the mouse brain. Nat Chem Biol 12 (10), 776-778.

34. Wang, W. et al. (2007) Genetically encoding unnatural amino acids for cellular and neuronal studies. Nat Neurosci 10 (8), 1063-72.

35. Zhu, S. et al. (2014) Genetically encoding a light switch in an ionotropic glutamate receptor reveals subunit-specific interfaces. Proc Natl Acad Sci U S A 111 (16), 6081-6.

36. Kang, J.Y. et al. (2013) In vivo expression of a light-activatable potassium channel using unnatural amino acids. Neuron 80 (2), 358-70. 
37. Zheng, Y. et al. (2017) Virus-Enabled Optimization and Delivery of the Genetic Machinery for Efficient Unnatural Amino Acid Mutagenesis in Mammalian Cells and Tissues. ACS Synth Biol 6 (1), 13-18.

38. Greiss, S. and Chin, J.W. (2011) Expanding the genetic code of an animal. J Am Chem Soc 133 (36), 14196-9.

39. Bianco, A. et al. (2012) Expanding the genetic code of Drosophila melanogaster. Nat Chem Biol 8 (9), 748-50.

40. Chen, Y. et al. (2017) Heritable expansion of the genetic code in mouse and zebrafish. Cell Res 27 (2), 294-297.

41. Han, S. et al. (2017) Expanding the genetic code of Mus musculus. Nat Commun 8, 14568.

42. Neumann-Staubitz, P. and Neumann, H. (2016) The use of unnatural amino acids to study and engineer protein function. Curr Opin Struct Biol 38, 119-28.

43. Isacoff, E.Y. and Larsson, H.P. (2013) Fluorescence Techniques for Studying Ion Channel Gating: VCF, FRET, and LRET. In Encyclopedia of Biophysics (1 edn) (Roberts, G.C.K. ed), pp. 816-824, Springer-Verlag Berlin Heidelberg.

44. Cohen, B.E. et al. (2002) Probing protein electrostatics with a synthetic fluorescent amino acid. Science 296 (5573), 1700-3.

45. Pantoja, R. et al. (2009) Single-molecule imaging of a fluorescent unnatural amino acid incorporated into nicotinic receptors. Biophys J 96 (1), 226-37.

46. Leisle, L. et al. (2016) Cellular encoding of Cy dyes for single-molecule imaging. eLife 5:19088.

47. Wang, J. et al. (2006) A genetically encoded fluorescent amino acid. J Am Chem Soc 128 (27), 8738-9.

48. Summerer, D. et al. (2006) A genetically encoded fluorescent amino acid. Proc Natl Acad Sci U S A 103 (26), 9785-9.

49. Liu, S. et al. (2015) $\mathrm{K}(+)$ preference at the NaK channel entrance revealed by fluorescence lifetime and anisotropy analysis of site-specifically incorporated (7hydroxycoumarin-4-yl)ethylglycine. Chem Commun (Camb) 51 (88), 15971-4.

50. Shen, B. et al. (2011) Genetically encoding unnatural amino acids in neural stem cells and optically reporting voltage-sensitive domain changes in differentiated neurons. Stem Cells $29(8), 1231-40$.

51. Chatterjee, A. et al. (2013) A genetically encoded fluorescent probe in mammalian cells. J Am Chem Soc 135 (34), 12540-3.

52. Lee, H.S. et al. (2009) Genetic incorporation of a small, environmentally sensitive, fluorescent probe into proteins in Saccharomyces cerevisiae. J Am Chem Soc 131 (36), 12921-3.

53. Mitchell, A.L. et al. (2017) A Unique Genetically Encoded FRET Pair in Mammalian Cells. Chembiochem 18 (6), 511-514. 
54. Aman, T.K. et al. (2016) Regulation of CNGA1 Channel Gating by Interactions with the Membrane. J Biol Chem 291 (19), 9939-47.

55. Dai, G. and Zagotta, W.N. (2017) Molecular mechanism of voltage-dependent potentiation of $\mathrm{KCNH}$ potassium channels. eLife 6:e26355.

56. Zagotta, W.N. et al. (2016) Measuring distances between TRPV1 and the plasma membrane using a noncanonical amino acid and transition metal ion FRET. J Gen Physiol 147 (2), 201-16.

57. Kalstrup, T. and Blunck, R. (2013) Dynamics of internal pore opening in $\mathrm{K}(\mathrm{V})$ channels probed by a fluorescent unnatural amino acid. Proc Natl Acad Sci U S A 110 (20), 8272-7.

58. Horne, A.J. and Fedida, D. (2009) Use of voltage clamp fluorimetry in understanding potassium channel gating: a review of Shaker fluorescence data. Can J Physiol Pharmacol 87 (6), 411-8.

59. Sakata, S. et al. (2016) Voltage-dependent motion of the catalytic region of voltagesensing phosphatase monitored by a fluorescent amino acid. Proc Natl Acad Sci U S A 113 (27), 7521-6.

60. Soh, M.S. et al. (2017) Probing the Structural Mechanism of Partial Agonism in Glycine Receptors Using the Fluorescent Artificial Amino Acid, ANAP. ACS Chem Biol 12 (3), 805813.

61. Pless, S.A. and Lynch, J.W. (2009) Ligand-specific conformational changes in the alpha1 glycine receptor ligand-binding domain. J Biol Chem 284 (23), 15847-56.

62. Wen, M. et al. (2015) Site-specific fluorescence spectrum detection and characterization of hASIC1a channels upon toxin mambalgin-1 binding in live mammalian cells. Chem Commun (Camb) 51 (38), 8153-6.

63. Miller, J.C. et al. (1998) Flash decaging of tyrosine sidechains in an ion channel. Neuron 20 (4), 619-24.

64. England, P.M. et al. (1997) Site-specific, photochemical proteolysis applied to ion channels in vivo. Proc Natl Acad Sci U S A 94 (20), 11025-30.

65. Philipson, K.D. et al. (2001) Incorporation of caged cysteine and caged tyrosine into a transmembrane segment of the nicotinic ACh receptor. Am J Physiol Cell Physiol 281 (1), C195-206.

66. Hanek, A.P. et al. (2010) Photochemical proteolysis of an unstructured linker of the GABAAR extracellular domain prevents GABA but not pentobarbital activation. Mol Pharmacol 78 (1), 29-35.

67. Lynagh, T. et al. (2017) A selectivity filter at the intracellular end of the acid-sensing ion channel pore. eLife 6:e24630.

68. Grunbeck, A. and Sakmar, T.P. (2013) Probing G protein-coupled receptor-ligand interactions with targeted photoactivatable cross-linkers. Biochemistry 52 (48), 8625-32.

69. Basle, E. et al. (2010) Protein chemical modification on endogenous amino acids. Chem Biol 17 (3), 213-27. 
70. Ye, S. et al. (2008) Site-specific incorporation of keto amino acids into functional G protein-coupled receptors using unnatural amino acid mutagenesis. J Biol Chem 283 (3), 1525-33.

71. Murray, C.I. et al. (2016) Unnatural amino acid photo-crosslinking of the IKs channel complex demonstrates a KCNE1:KCNQ1 stoichiometry of up to 4:4. eLife 5:e11815.

72. Westhoff, M. et al. (2017) Photo-Cross-Linking of IKs Demonstrates State-Dependent Interactions between KCNE1 and KCNQ1. Biophys J 113 (2), 415-425.

73. Kumar, J. and Mayer, M.L. (2013) Functional insights from glutamate receptor ion channel structures. Annu Rev Physiol 75, 313-37.

74. Baranovic, J. and Plested, A.J. (2016) How to build the fastest receptor on earth. Biol Chem 397 (3), 195-205.

75. Klippenstein, V. et al. (2014) Photoinactivation of glutamate receptors by genetically encoded unnatural amino acids. J Neurosci 34 (3), 980-91.

76. Zhu, S. and Paoletti, P. (2015) Allosteric modulators of NMDA receptors: multiple sites and mechanisms. Curr Opin Pharmacol 20, 14-23.

77. Tian, M. and Ye, S. (2016) Allosteric regulation in NMDA receptors revealed by the genetically encoded photo-cross-linkers. Sci Rep 6, 34751.

78. Ye, S. et al. (2013) Expanding the genetic code in Xenopus laevis oocytes. Chembiochem 14 (2), 230-5.

79. Hoppmann, C. et al. (2014) Genetically encoding photoswitchable click amino acids in Escherichia coli and mammalian cells. Angew Chem Int Ed Engl 53 (15), 3932-6.

80. Klippenstein, V. et al. (2017) Optocontrol of glutamate receptor activity by single sidechain photoisomerization. eLife 6:e25808.

81. Hoppmann, C. et al. (2015) In Situ Formation of an Azo Bridge on Proteins Controllable by Visible Light. J Am Chem Soc 137 (35), 11218-21.

82. Wang, K. et al. (2014) Optimized orthogonal translation of unnatural amino acids enables spontaneous protein double-labelling and FRET. Nat Chem 6 (5), 393-403.

83. Sandoz, G. et al. (2012) Optical control of endogenous proteins with a photoswitchable conditional subunit reveals a role for TREK1 in GABA(B) signaling. Neuron 74 (6), 1005-14.

84. Kienzler, M.A. et al. (2013) A red-shifted, fast-relaxing azobenzene photoswitch for visible light control of an ionotropic glutamate receptor. J Am Chem Soc 135 (47), 17683-6.

85. Samanta, S. et al. (2013) Photoswitching azo compounds in vivo with red light. J Am Chem Soc 135 (26), 9777-84.

86. Kalstrup, T. and Blunck, R. (2015) Reinitiation at non-canonical start codons leads to leak expression when incorporating unnatural amino acids. Sci Rep 5, 11866.

87. Karakas, E. and Furukawa, H. (2014) Crystal structure of a heterotetrameric NMDA receptor ion channel. Science 344 (6187), 992-7. 
88. Grunbeck, A. et al. (2011) Mapping the ligand-binding site on a G protein-coupled receptor (GPCR) using genetically encoded photocrosslinkers. Biochemistry 50 (17), 3411-3.

89. Ahern, C.A. and Kobertz, W.R. (2009) Chemical tools for $\mathrm{K}(+)$ channel biology. Biochemistry 48 (3), 517-26. 


\section{TABLE \& FIGURE LEGENDS}

Table 1: Methodologies to confer light-sensitivity to an ion channel.

\section{Table 2: Overview of commonalities and differences between two light-sensitive probes - UAAs and PTLs.}

\section{Figure 1: Genetically encoding light-sensitive UAAs into ion channels.}

The UAA genetic encoding methodology, as performed for mammalian cell lines, is represented schematically. (a) Vectors carrying genes for an ion channel of interest (light blue) and an orthogonal suppressor tRNA (orange) / aminoacyl synthetase (dark blue) pair are introduced into the cell by means of transient transfection. The amber stop codon (TAG, red) replaces a native codon at a permissive site within the sequence of the ion channel gene. (b) The light-sensitive UAA (purple asterisks) is supplemented into the cellular growth medium and spontaneously enters the cell, presumably through amino acid transporters (grey). (c) Within the cell, the orthogonal synthetase specifically aminoacylates the suppressor tRNA with the UAA, a catalytic reaction driven by ATP. (d) The UAA-carrying tRNA, which contains a CUA anticodon, enters the ribosomal machinery to incorporate the UAA in response to the complementary amber codon on the ion channel mRNA (black). (e) Once relieved from the charge at the ribosome, the tRNA can be reused for further UAAaminoacylation by the cognate synthetase. ( $\boldsymbol{f})$ The full-length polypeptide chain (here, exemplarily shown for two NMDAR subunits, PDB 4PE5 [87]; light blue), site-specifically carrying the UAA, undergoes folding and assembly into a functioning ion channel. $(\boldsymbol{g})$ The newly formed membrane protein migrates to its assigned location (e.g. the cell surface) to selectively conduct ions (yellow), thus contributing to cellular functions.

Figure 2: Fluorescent UAAs (fUAAs) to track ion channel structural dynamics.

(A) Principle of VCF. An environment-sensitive fUAA (yellow asterisk) is genetically encoded at a specific location of an ion channel. Fluorescence of this UAA (blue thunderbolt) depends on the conformational state of the ion channel and can be correlated to the opening state of the channel through current measurements. (B) Chemical structures of fUAAs incorporated in ion channels using in vitro amino-acylated tRNAs. (C) Chemical structures of fUAAs, for which a bio-orthogonal tRNA/synthetase pair has been developed. (D) Motions of the intra- 
and extra-cellular ends of the S4 segment, simultaneously recorded with gating currents (induced by S4 motion) in Shaker [57]. Upper panel: Schematic representation of Shaker. Only two of the four subunits are represented. In ref [57], Anap (red star) was introduced at the intra-cellular end of S1 (V234), close to S4, while a cysteine in the S3-S4 loop (A359) was labeled with TMR (green asterisks). Lower panel: Gating currents (I) and fluorescence responses $\left(F_{\text {Anap }}\right.$ and $\left.F_{\text {TMR }}\right)$ of TMR-labeled Shaker-V234Anap-A359C channels. From ref [57].

\section{Figure 3: Examples of optical ion channel modulation by light-sensitive UAAs.}

Ion channels are illustrated in blue. (A) Incorporation of a caged UAA (5-branch asterisk), here a caged cysteine, in the pore of a VGIC (e.g. PIRK [36]) hinders the conductance of ions (yellow). UAA photolysis by UV light (purple thunderbolt) releases the cage (8-branch asterisk) and restores current flow. (B) Chemical reaction of $\mathrm{Cmn}$ photolysis. (C) UV-induced photo-crosslinking of a VGIC to an auxiliary subunit (green). (D) Chemical reactions of the covalent photo-crosslinking by BzF and AzF following UV illumination. (E) Photo-inactivation by intra-receptor UV-crosslinking of a LGIC (e.g. an iGluR). Photo-crosslinking UAAs, placed at key moving interface sites, physically link adjacent subunits. (F) Left, representative time course of inactivation of the homomeric GluA2 AMPA receptor (with BzF at the S729 site), induced by photo-crosslinking of subunits, following application of UV pulses (violet bars) of different durations (red and green circles). Right, example traces recorded before (black) and at the end (red and green) of the two UV protocols of the left panel, highlighting UV-mediated receptor silencing. From ref [75]. (G) Reversible photo-modulation of ion channel activity. Photo-switchable UAAs, placed at key modulatory sites, can rapidly turn on or turn off receptor activity by toggling between a cis (purple asterisk) and a trans configuration (blue asterisk), induced by UV and blue/green range wavelengths (purple and blue thunderbolts), respectively. (H) Chemical structures of the cis and trans photoisomers of PSAA, a photoswitchable UAA. (I) Representative current trace of GluN1/GluN2A NMDARs carrying PSAA at GluN1-P532 under illumination by UV and blue light, demonstrating reversible photomodulation. From ref [80]. 


\section{BOX 1}

\section{COOKBOOK: ENGINEERING LIGHT-SENSITIVE ION CHANNELS IN MAMMALIAN CELL}

LINES

Overall, genetically encoded UAAs can be introduced into any ion channel of interest, if certain technical and strategic proceedings are respected. Most of the mentioned lightsensitive UAAs can be obtained commercially and their corresponding tRNA/synthetase pairs are usually available on request from groups, who have evolved those.

The introduction of UAAs calls for high-level molecular precision. Hence, protein engineering using UAAs benefits from existing structural and molecular information, facilitating technical processes including mutagenesis, experimental design, and data interpretation. Prior investigating novel, poorly resolved domains, introduce the UAA at well characterized ion channel regulatory sites to gain first broad ideas about the efficiency of UAA incorporation, the resulting ion channel functional properties, and their behavior following optical modulation. Generally, incorporating UAAs in large proteins such as ion channels involves a screening process to identify strong candidate positions, assigned by minor functional side-effects following UAA introduction per se (ion channel carrying UAAs should desirably show wild-type-like biophysical properties) and major functional impacts upon light-stimulation. Indeed, even if UAAs produce only minor structural perturbations, since only one side-chain within the polypeptide sequence is altered, these are not always subtle. Light-sensitive UAAs, usually of aromatic nature, are bulkier in size compared to natural amino acids and may therefore induce significant perturbation of channel function that could interfere with the optically induced effects. Furthermore, since photo-modulation measurements often require long-lasting recordings, the UAA has to be stable within the protein, with no unspecific side-effects in the absence of light, as for example observed for AzF [80, 88].

Usually, the degree of photo-effects depends on the duration and intensity of light, with faster photo-modulation rates following longer and stronger illumination (see, for example, ref [80]). To ensure that the maximal photo-effect is obtained, use a strong source of light, such as high-power LEDs, at a precise illumination wavelength in accordance to the UAA absorbance spectrum.

Importantly, verify that truncated ion channel versions, due to a premature termination of protein translation or a shift of its initiation [78, 86], do not interfere with the function of fulllength ion channels or even induce false-positive effects. Also, evaluate the degree of unspecific read-through by endogenous amino acids, which can be significant, in a 
standardized manner by control experiments in the absence of the UAA (see, for instance, ref [78]; discussed in ref [89]). Lastly, consider the multimeric nature of ion channels in the interpretation of results. Depending on the subunit composition, an ion channel may contain multiple UAA copies, thus potentially producing complex photo-effects upon light-stimulation. Particularly, this is important when determining the kinetics of the photo-effects. 


\section{GLOSSARY}

Azobenzene moiety: Azobenzene moieties undergo reversible toggling between two photoisomerizable states in dependence of light illumination - a compact cis- (usually upon exposure to UV light) and a stretched trans-configuration (upon application of light in the blue/green range). The absorbance spectrum is variable in dependence of adjoined chemical modifications. Because of their synthetic tractability, high quantum yield, stability, and biocompatibility, azobenzene moieties are currently the most commonly used photoswitches.

Bio-orthogonality: Specifically engineered tRNA/synthetase pairs must be bio-orthogonal to avoid crosstalk with the native biosynthetic machinery. An orthogonal tRNA must not be aminoacylated by any endogenous tRNA-synthetase and an orthogonal synthetase must not aminoacylate any endogenous tRNA with endogenous amino acids. When incorporating UAAs, bio-orthogonality can be checked using proper control experiments.

FRET: Förster Resonance Energy Transfer is a highly sensitive technique that allows the transfer of energy between two chromophores, a donor and an acceptor. The efficiency of energy transfer depends on the distance and orientation between the two chromophores. FRET measurements are therefore powerful tools to study distance changes between two domains in real time, as occurs during ion channel gating.

Genetic code expansion: Genetic code expansion allows introducing amino acids (unnatural amino acids, UAAs) that are not specified by the standard genetic code. Sitespecific incorporation of UAAs into proteins expressed in living cells occurs by suppression of nonsense codons (stop codons) by UAA-aminoacylated tRNAs. Traditionally, amber (TAG) stop codons are used. Efficient suppression of an amber codon results in a functional fulllength protein. UAAs carry side-chains with diverse physicochemical properties, not available in the 20 natural amino acids, thus adding novel functionalities into proteins. They can be introduced in any kind of protein, regardless of its type, size, or location.

VCF: Voltage-clamp fluorometry is a technique to simultaneously measure ion channel electrical activity through electrophysiology and monitor local molecular rearrangements through spectroscopic approaches such as fluorescence quenching or FRET. VCF based on fluorescence relies on the sensitivity of specific fluorophores to the chemical properties of their local environment (polarity and presence of endogenous quenching groups). Conformational rearrangements, such as during ion channel gating, provoke changes in the 
environment of the fluorophore, thus instantaneously changing its emission spectrum or fluorescence quantum yield. VCF is a powerful method to correlate ion channel structure and function in real-time. 


\section{OUTSTANDING QUESTIONS}

- Studying ion channels in vivo is essential for understanding their physiological and pathological roles. Transgenic animals with an expanded genetic code open possibilities to manipulate specific ion channel populations in whole tissues or organisms. Challenges to address are numerous. For example, can chronic UAA delivery be achieved? How prominent are off-target UAA effects? Can 'faulty' ion channel subunits, including truncated versions or those carrying endogenous amino acids instead of the UAA, be minimized?

- fUAAs are powerful tools to investigate structural mechanisms of recombinant ion channels. Whether this technology can be expanded to more native situations remains unclear. fUAAs in vivo have the potential to inform on how the cell microenvironment influences channel gating and what the underlying mechanisms are. For enhanced biocompatibility, genetically encoded fUAAs with better spectral properties (such as stimulation by red-shifted wavelengths) are needed.

- The occurrence of specific ion channel subpopulations is dynamic, depending on the tissue, cell type, developmental stage, and subcellular location. At a given time and location, what is the ion channel diversity landscape and how is it dynamically regulated? Optogenetic pharmacology provides new means to address these questions with unprecedented precision.

- UAAs can in principle be inserted anywhere in the protein, allowing control of channel activity at multiple channel locations. Introducing light-sensitive UAAs in cytoplasmic regions of an ion channel should help interrogate how it interacts with intracellular partners and how dynamic these interactions are.

- The vast investigation of ion channels and their interacting partners in their native environment is essential to define biological targets in drug design. Defining novel and potent medication strategies that reach beyond the structural level of proteins could be key to treat diseases linked to dysfunction in ion channel signaling. 


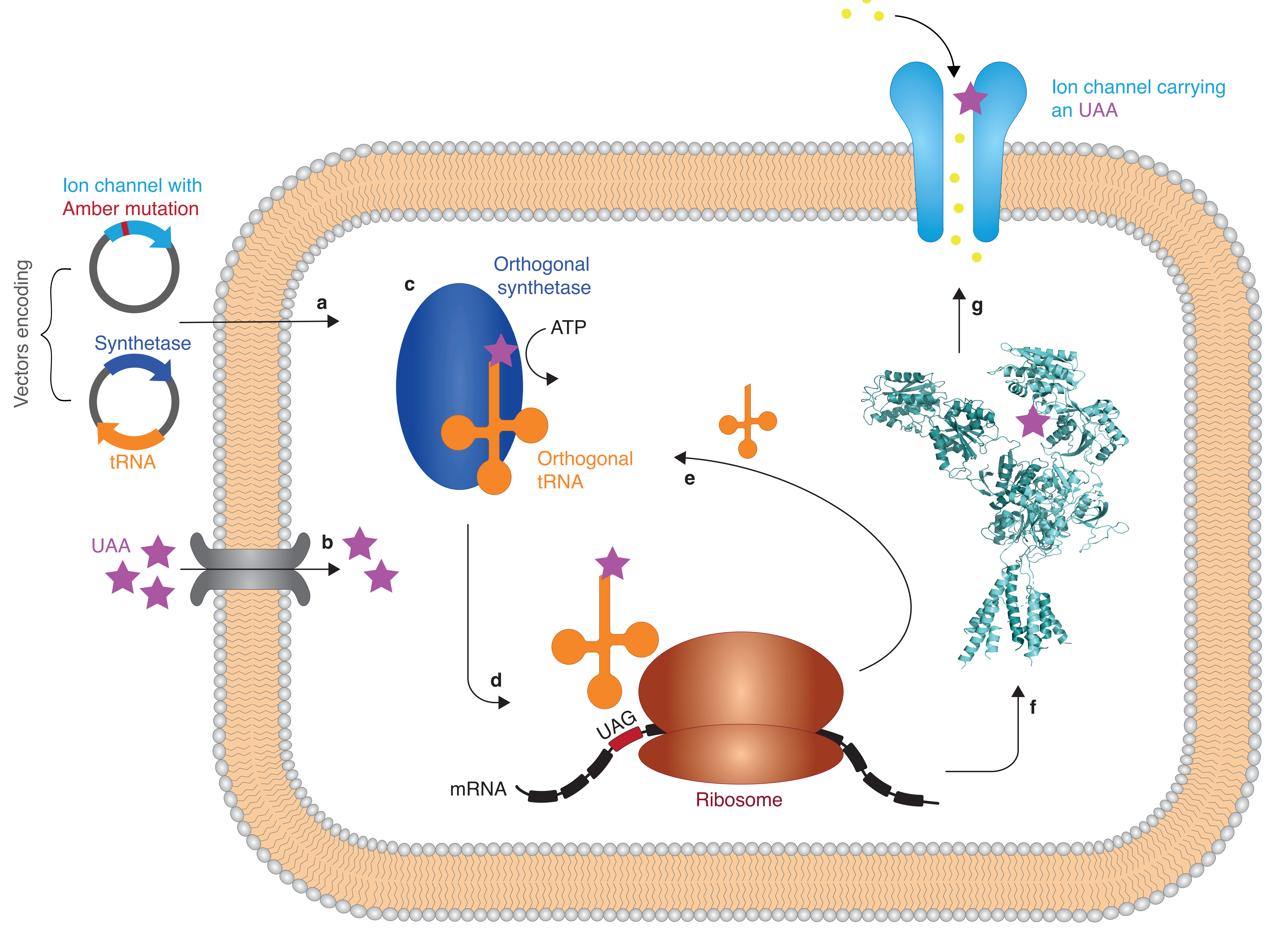




\section{A}
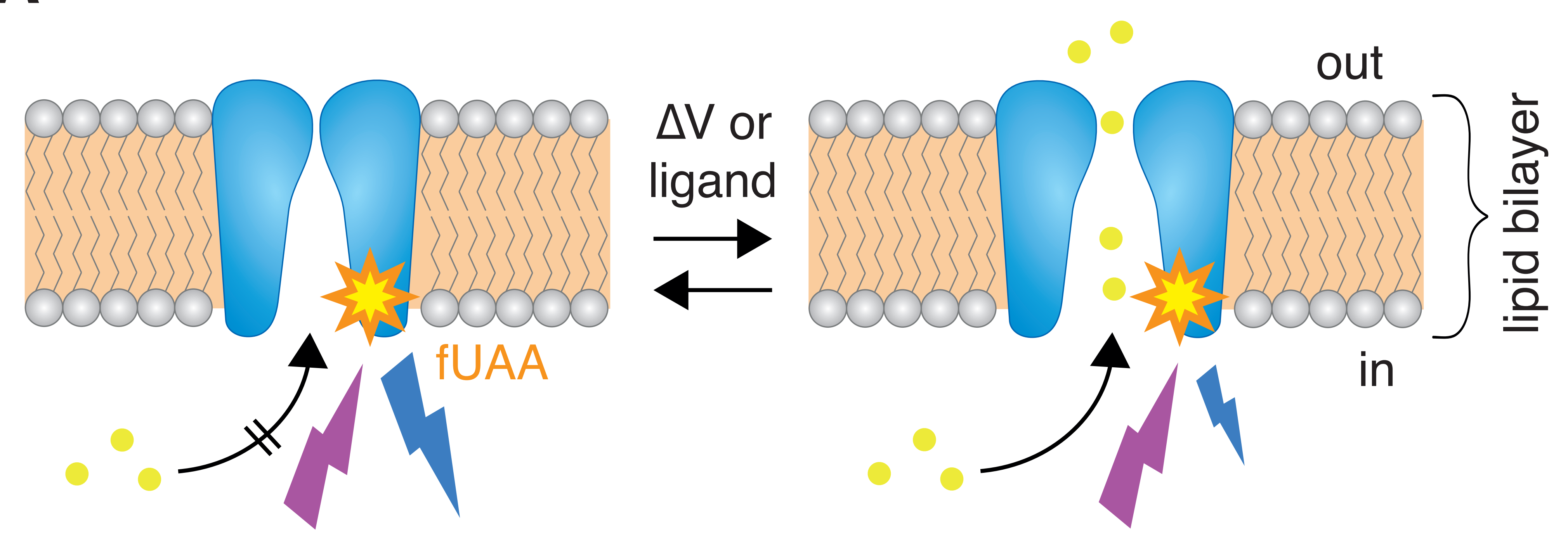

B
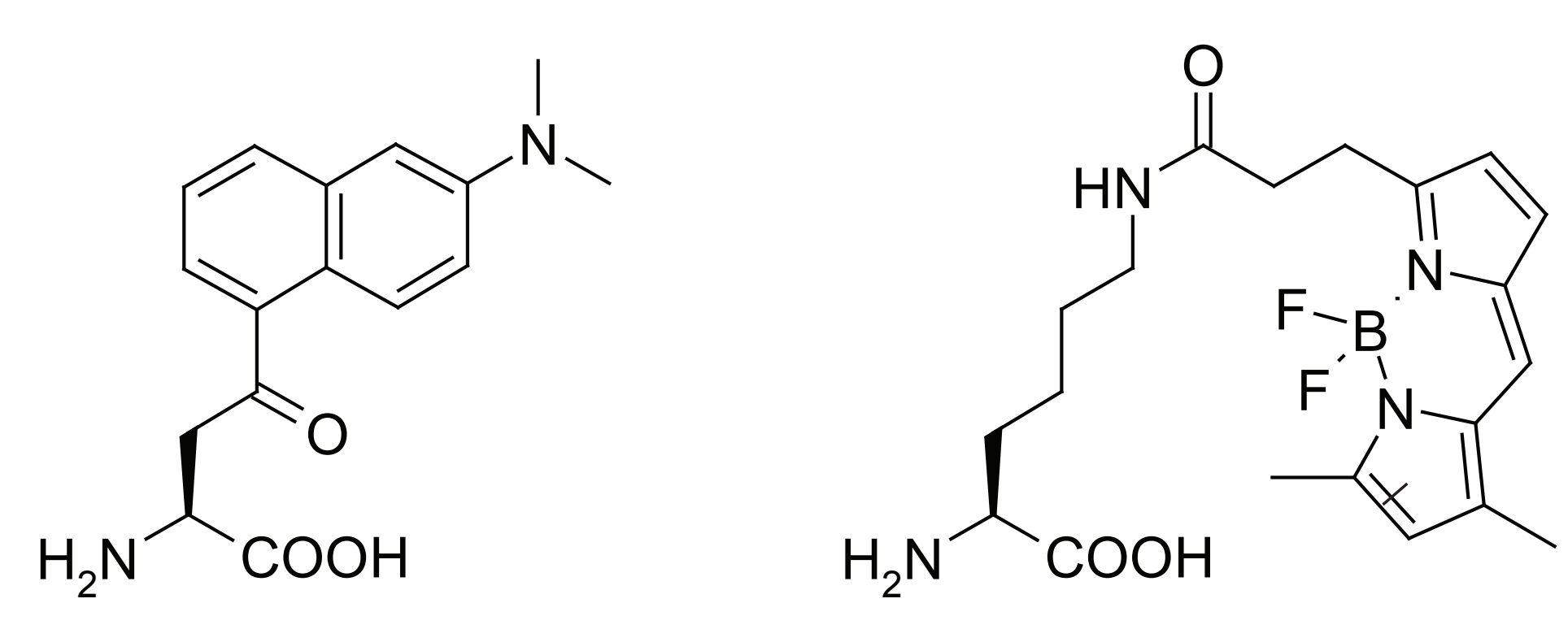

DAN-Alanine

BODIPYFL-Lysine

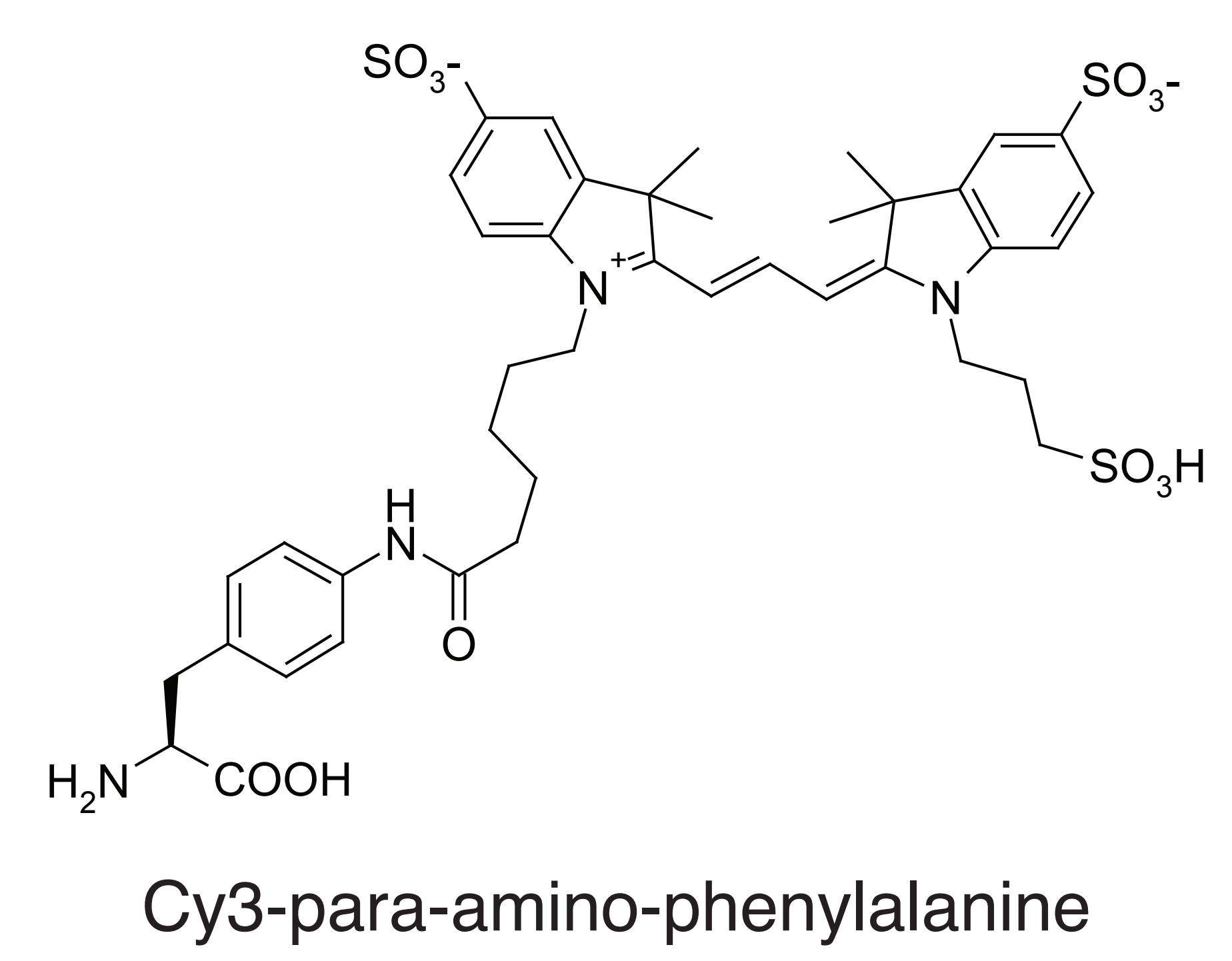

C
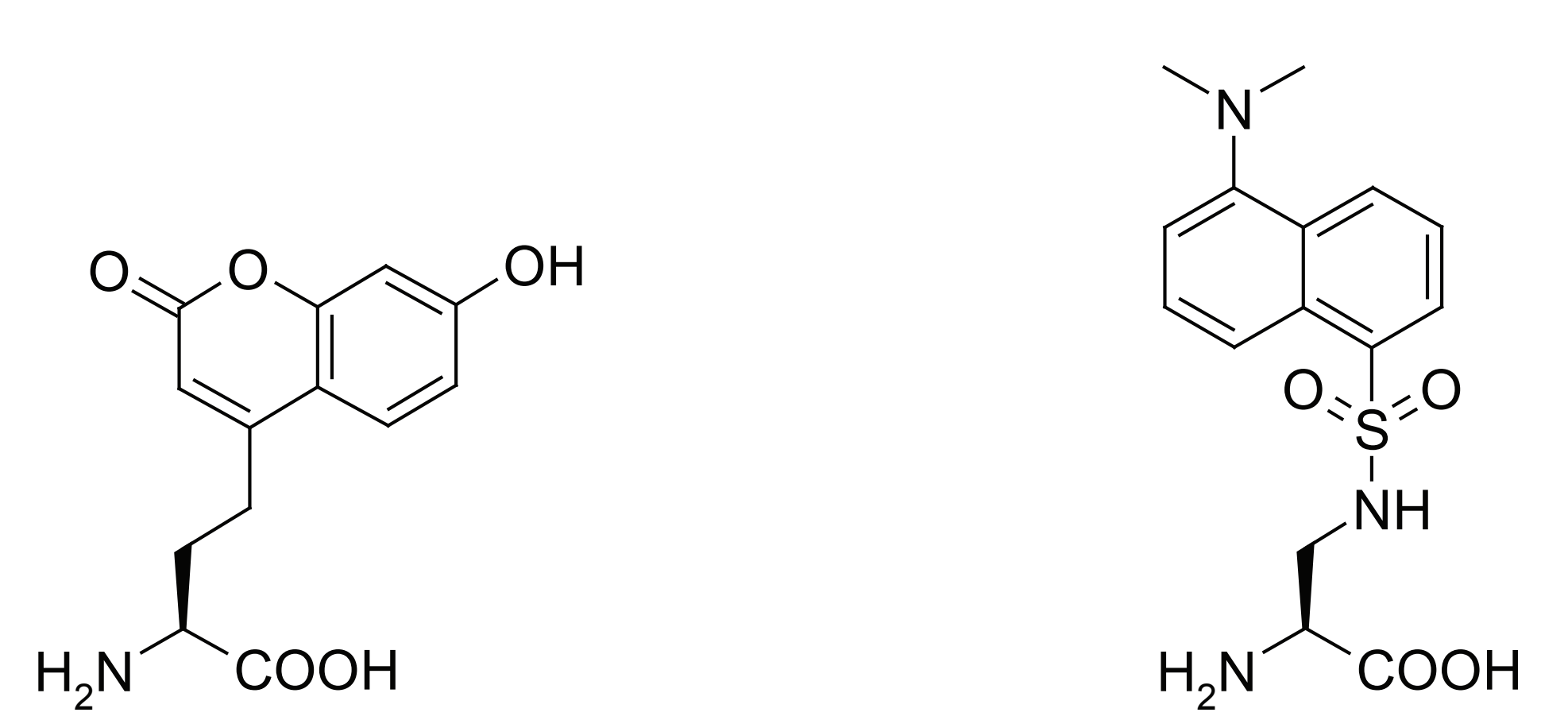

(7-hydroxycoumarin-4-yl) ethylglycine
D
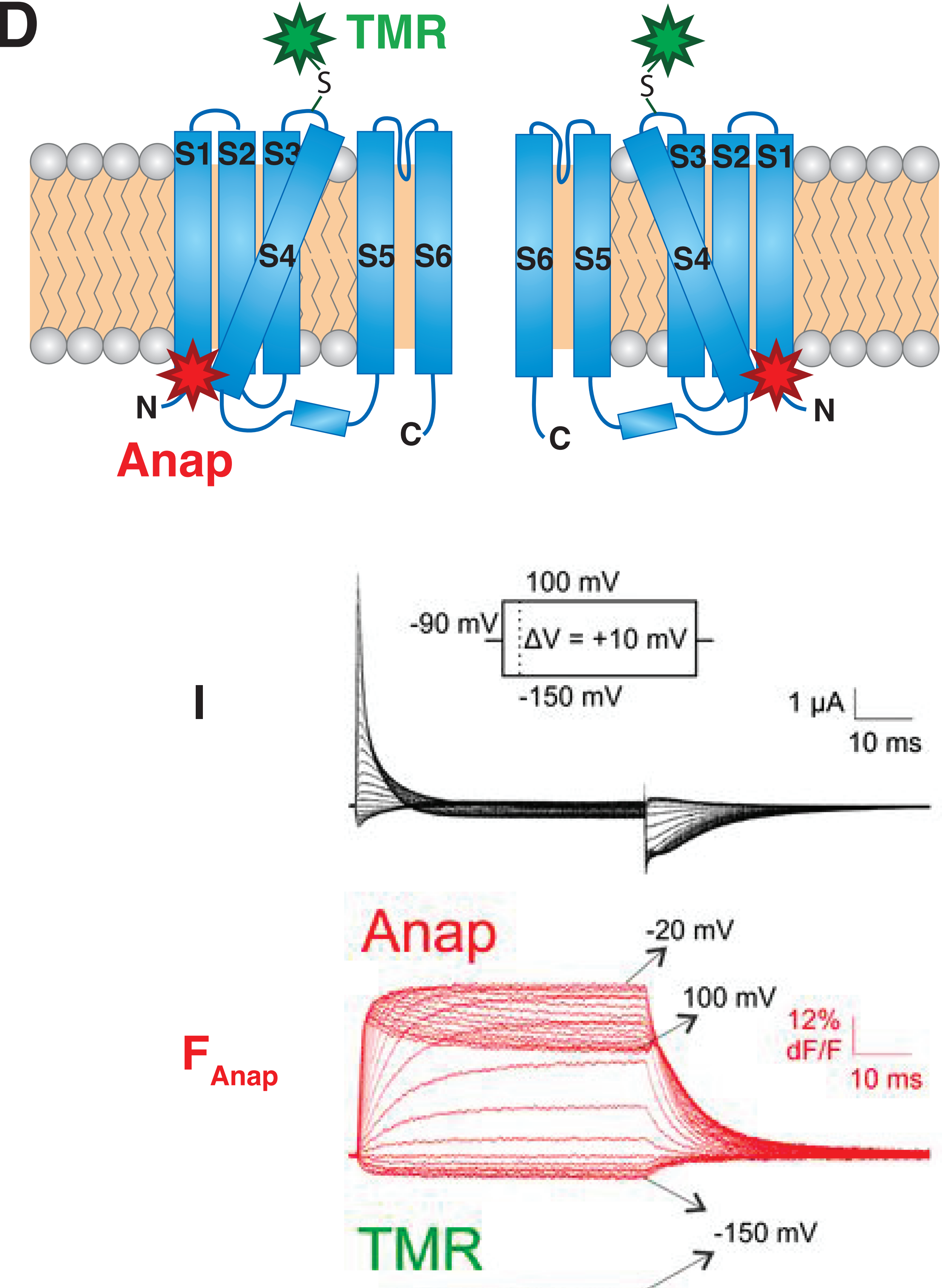

$\mathbf{F}_{\text {TMR }}$
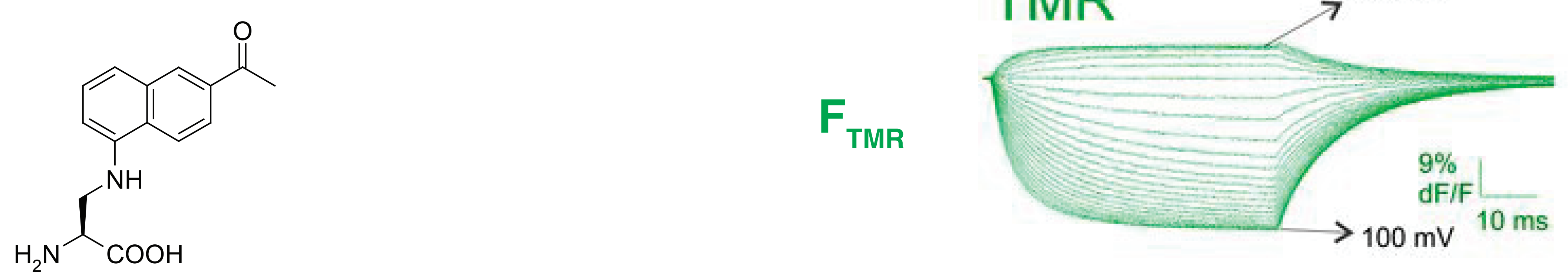
A Photo-uncaging

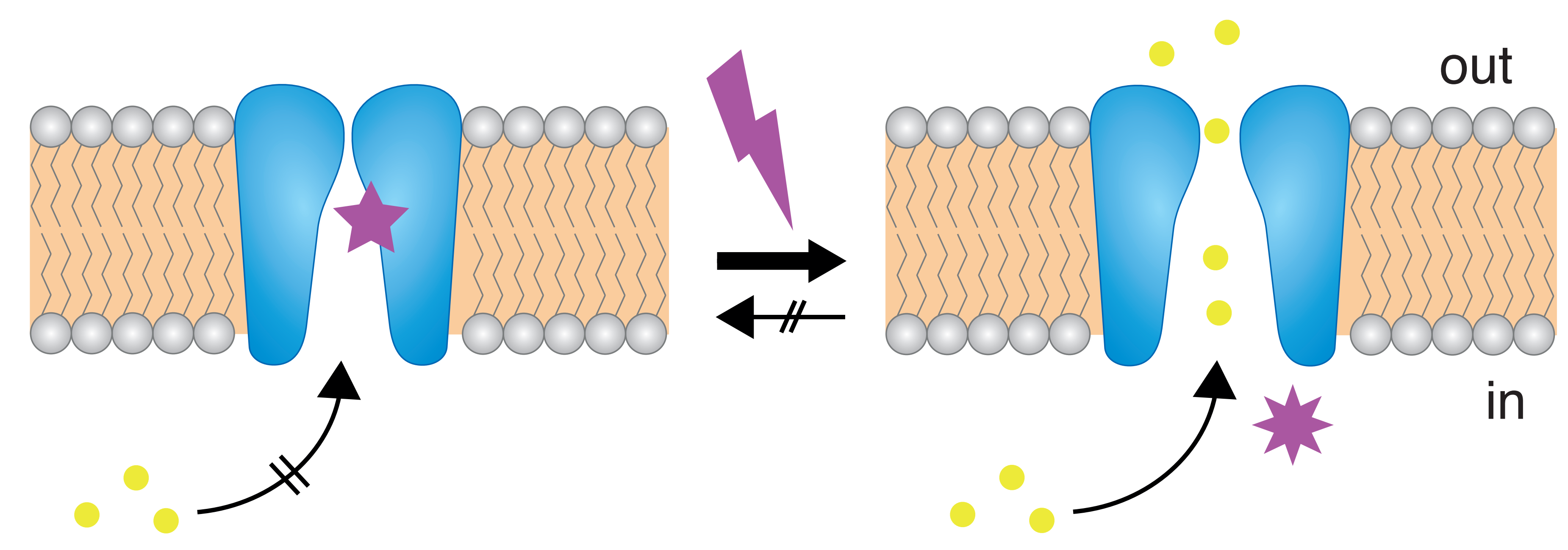

C Photo-crosslinking to interacting proteins

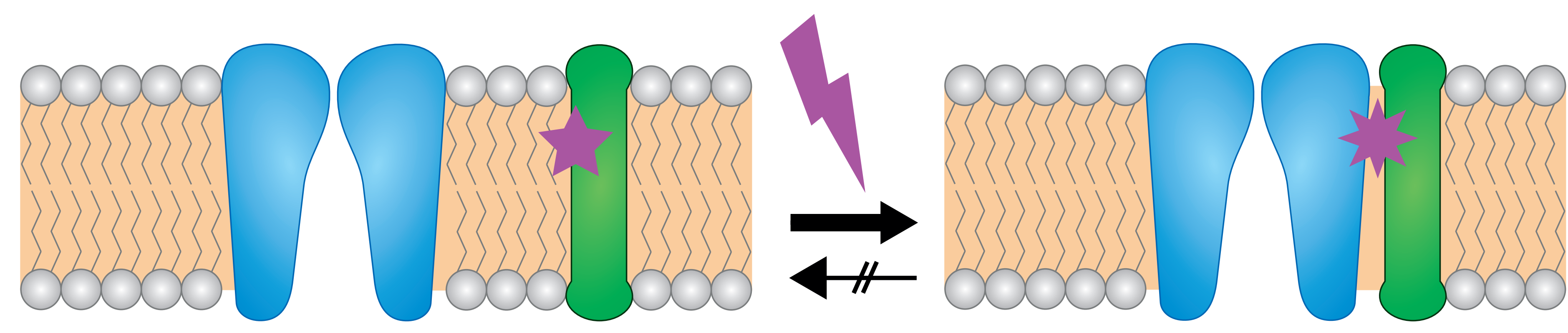

E Photo-crosslinking between subunits

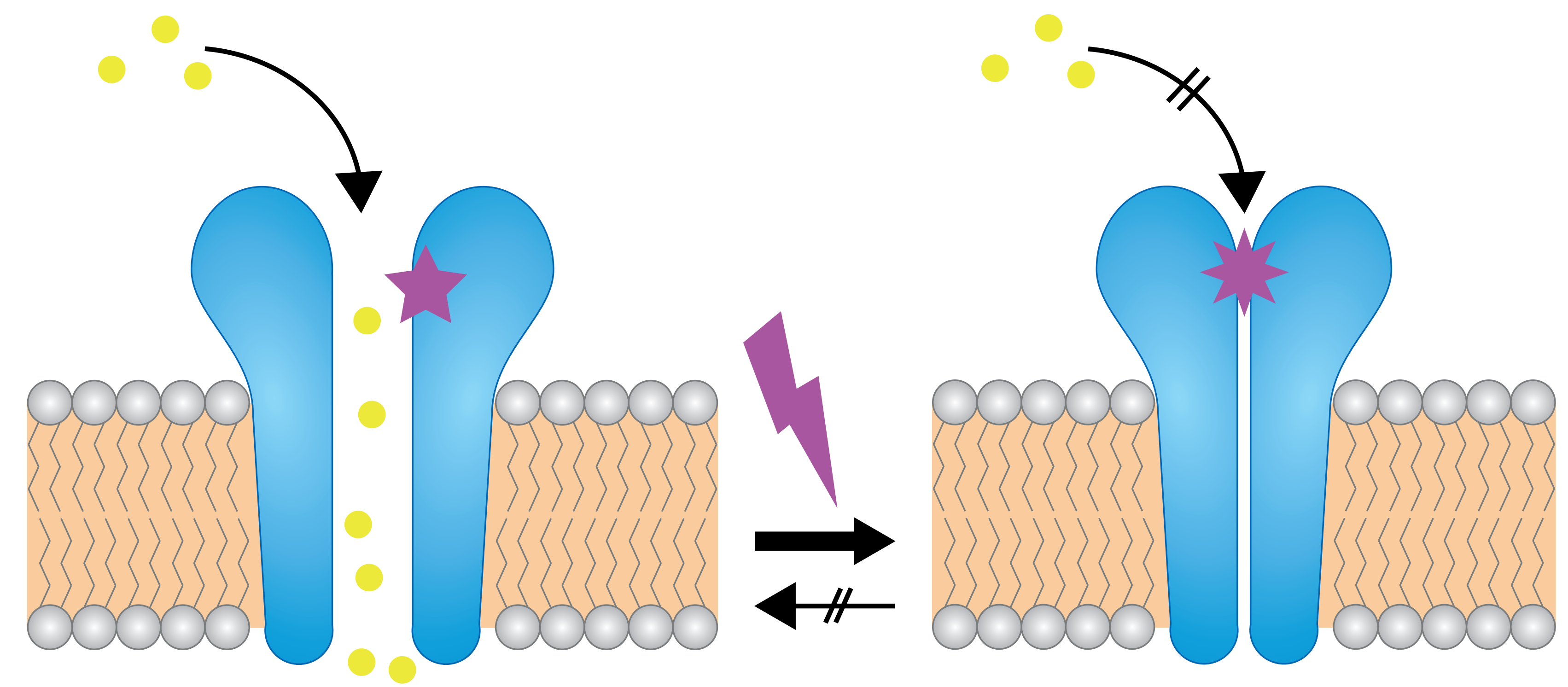

G Photo-switching

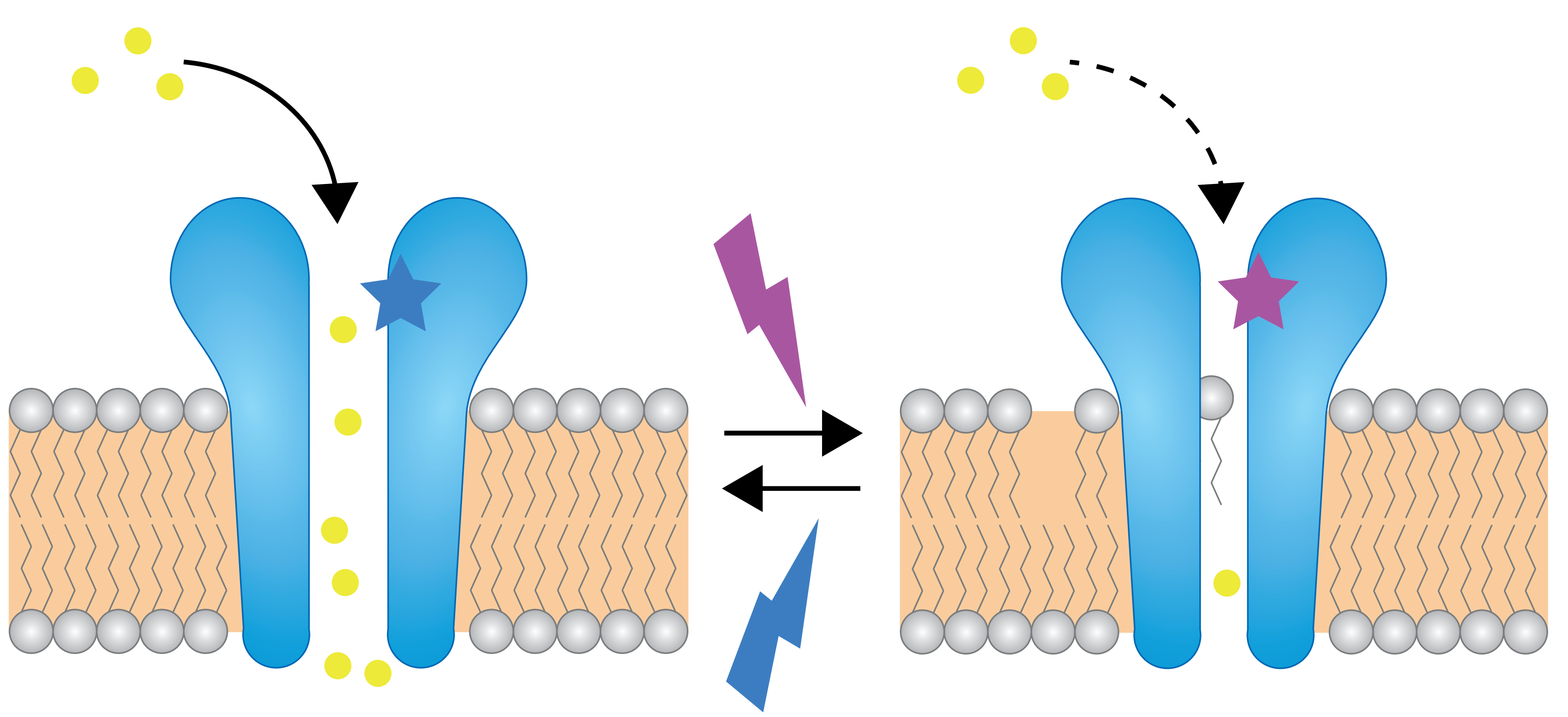

B

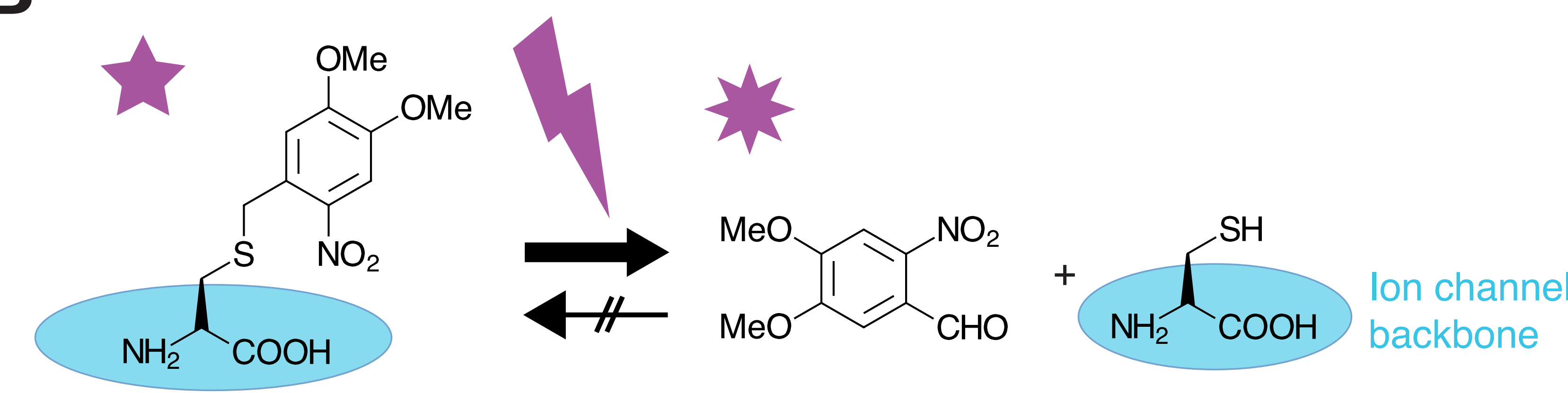

Cmn

D
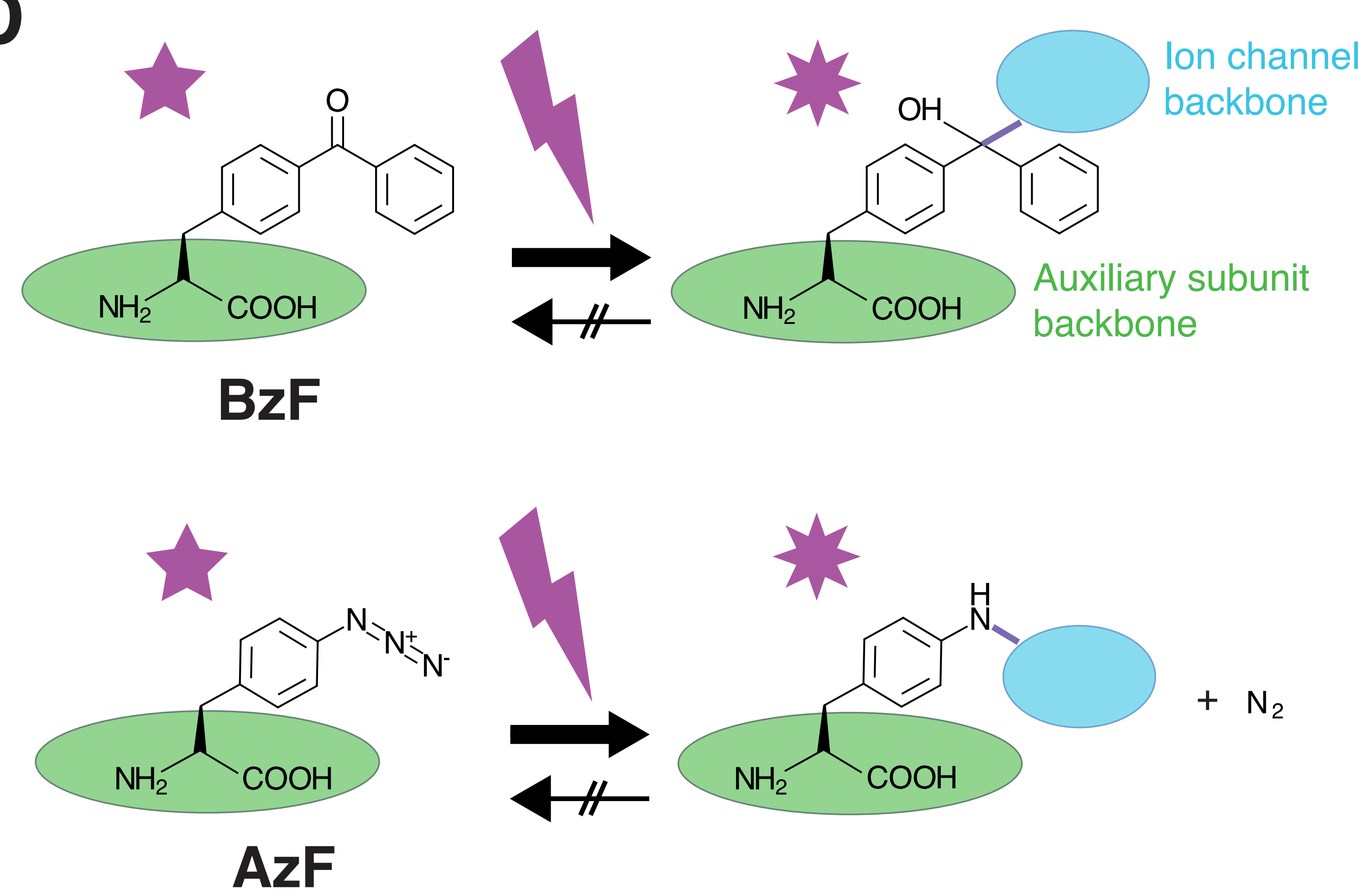

$F_{\text {GluA2 S729BzF }}$
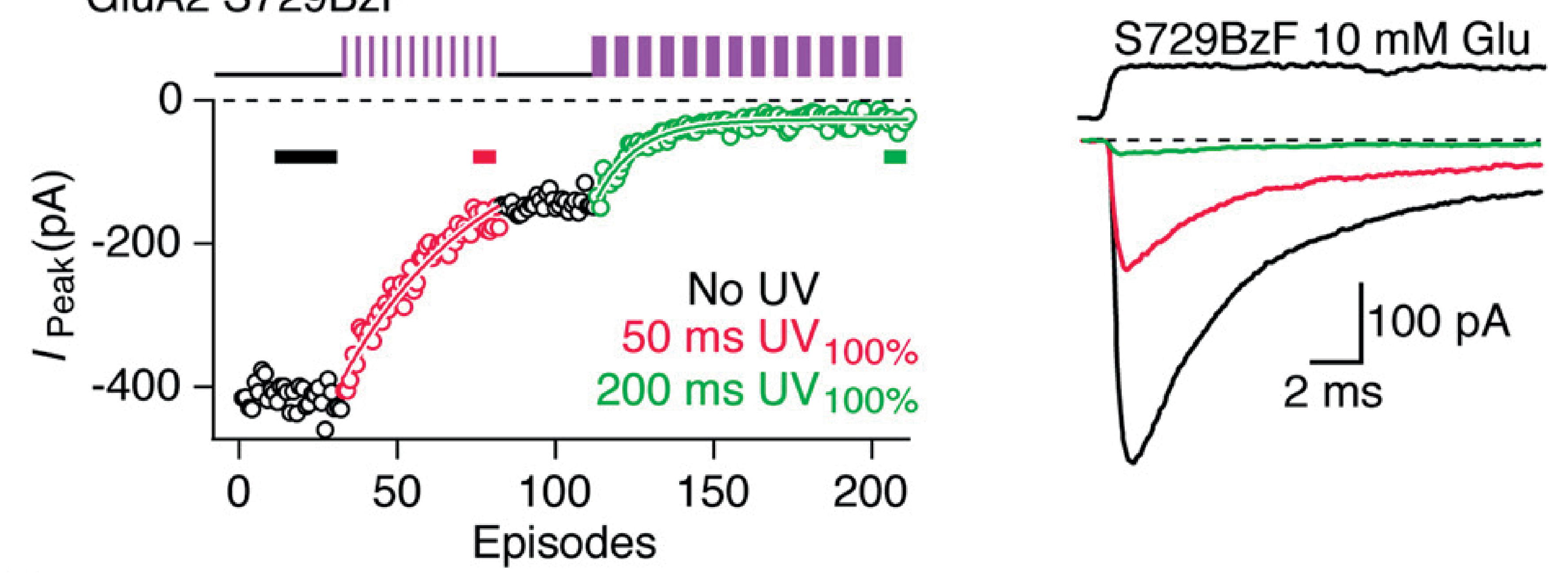

H

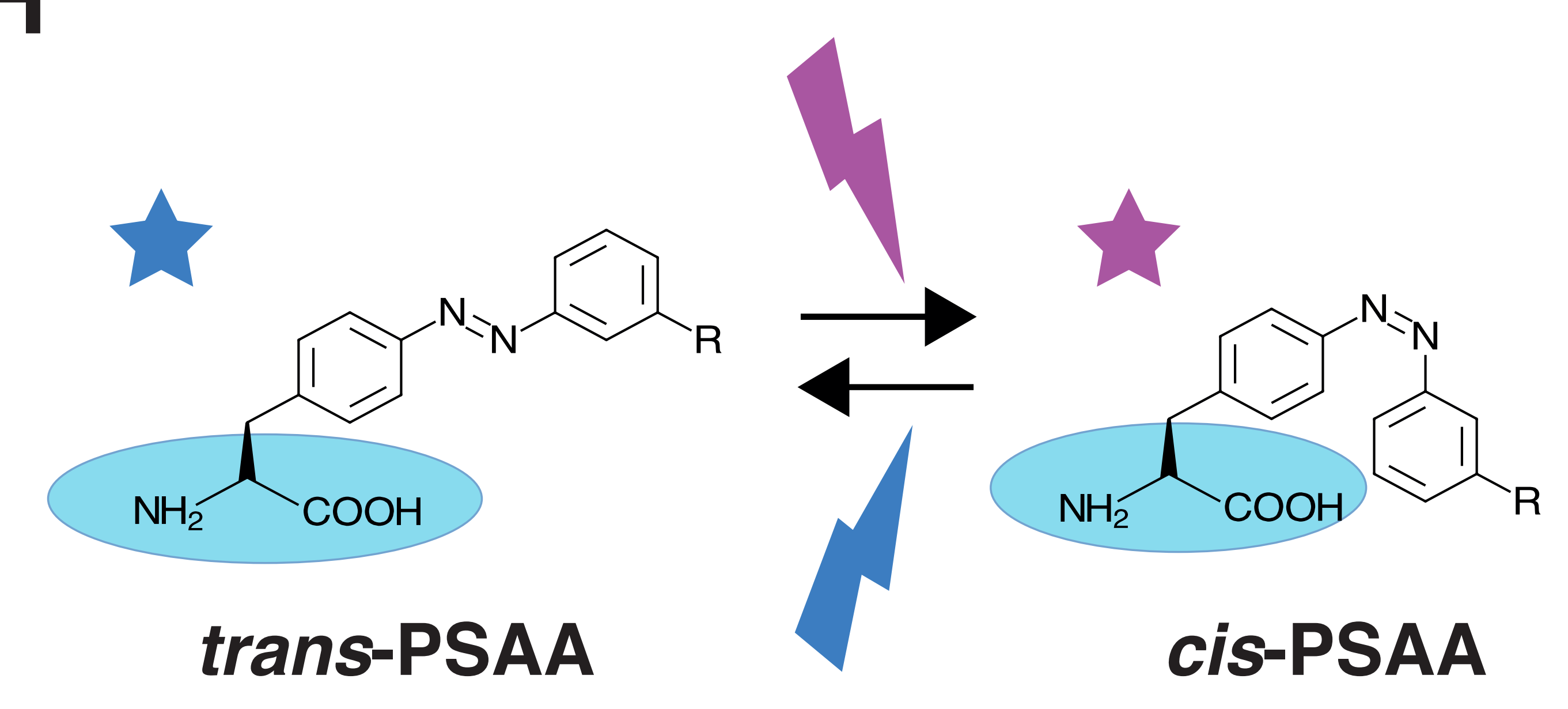

GluN1-P532PSAA

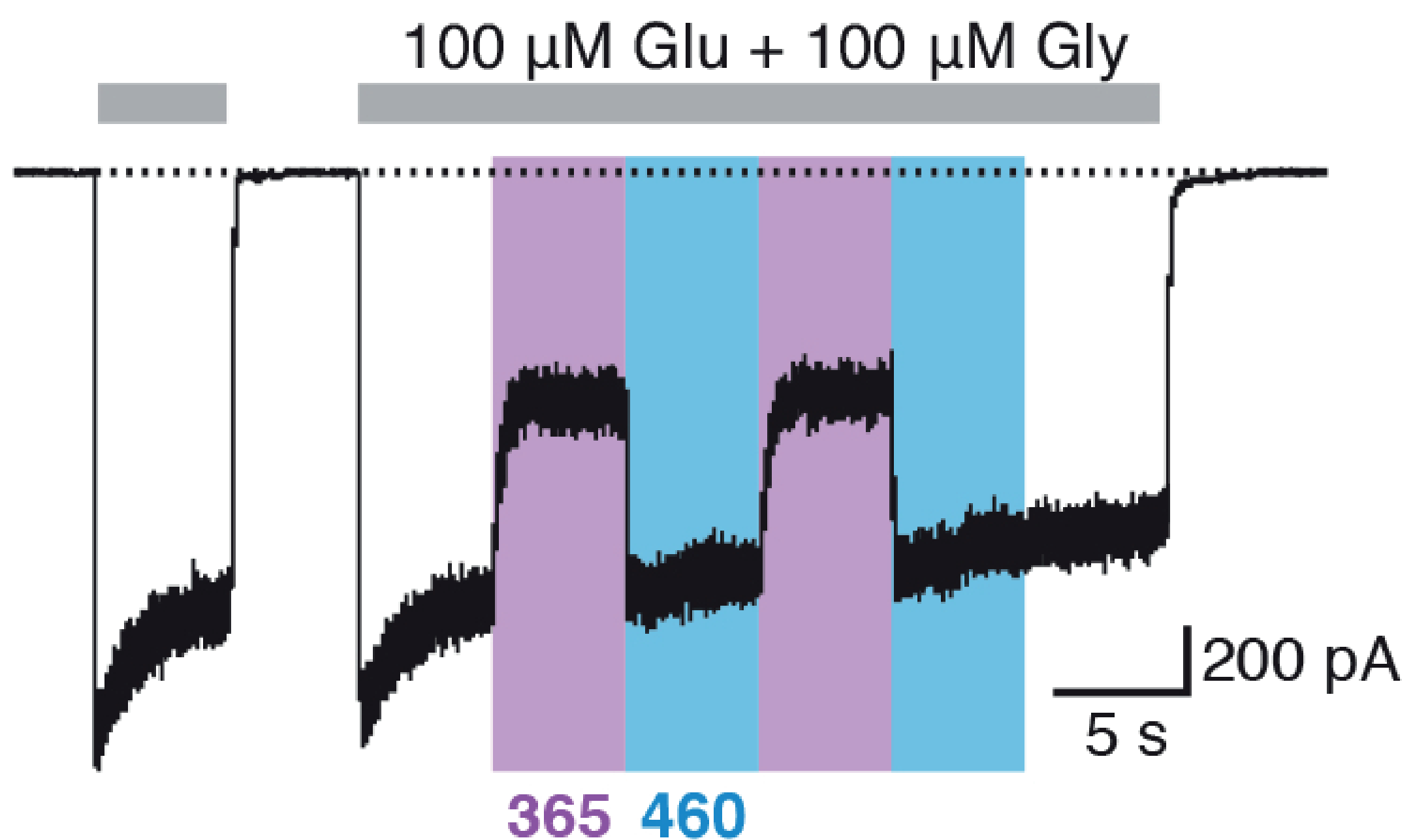




\section{DOMAIN}

\section{OPTOPHARMACOLOGY}

\section{OPTOGENETIC PHARMACOLOGY}

\section{OPTICAL MONITORING} (including VCF / PCF)

\section{INTRODUCTION OF} LIGHT-SENSITIVITY

\section{DIFFUSIBLE LIGANDS}

No genetic modification of the target protein

\section{TETHERED LIGANDS}

Post-translational labelling of the genetically modified target protein (cysteine mutation)

\section{CONDITIONS}

\section{PHOTO-SENSITIVE LIGANDS}

- caged compounds (irreversible)

example: MNI-glutamate

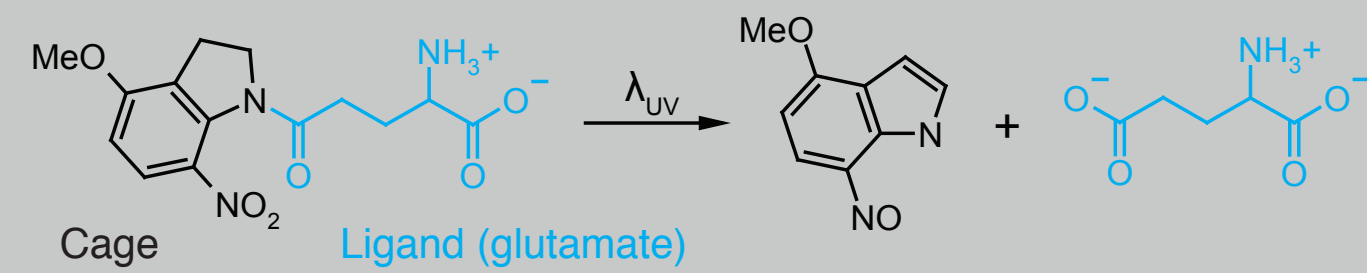

- azobenzene-based photo-switches (reversible)

example: 4-GluAzo

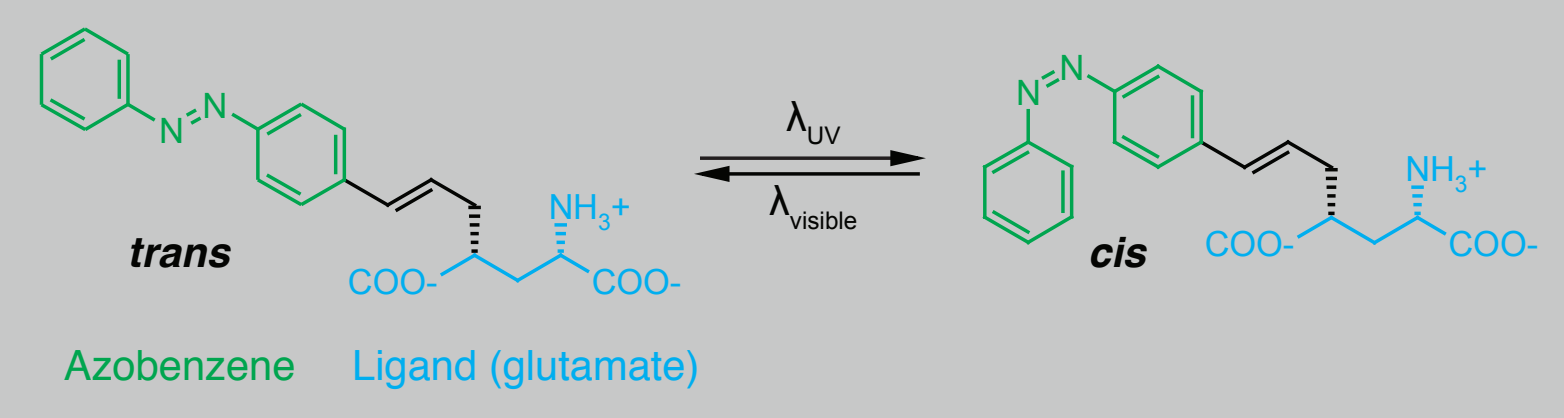

PHOTO-SENSITIVE TETHERED LIGANDS (PTLS)

- cysteine- and photo-reactive compounds (irreversible)

example: Benzophenone-4-carboxamidocysteine methanethiosulfonate (BPMTS)

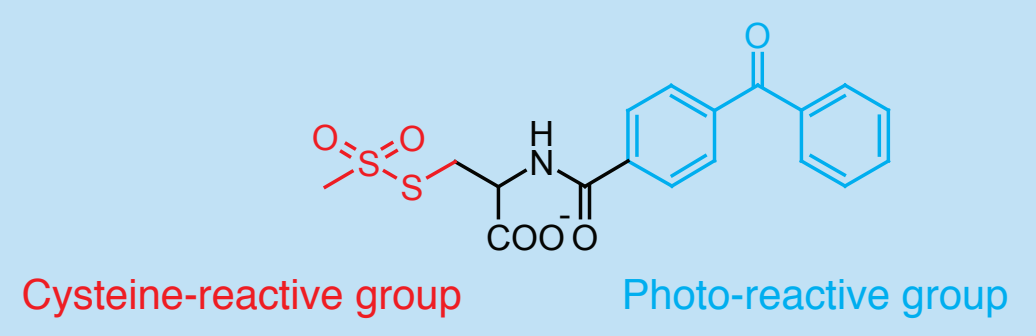

- cysteine-reactive, azobenzene-based photo-switches (reversible)

example: Maleimide-Azobenzene-Glutamate (MAG)

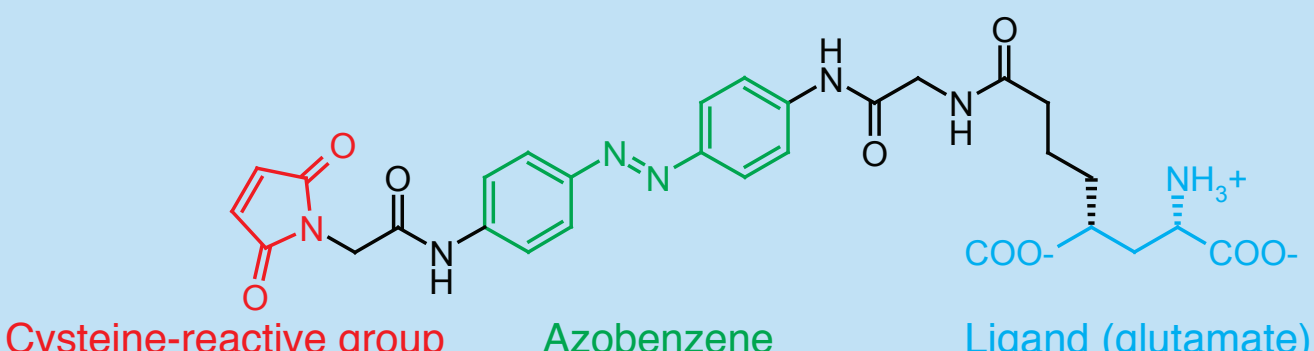

Light-sensitivity is directly conferred by the UAA (see Figure 3)

\section{Genetic incorporation}

\section{TETHERED FLUOROPHORES}

- cysteine- and tag-reactive compounds

\section{TETHERED LIGANDS} AS BIOPHYSICAL PROBES

Post-translational labelling of the genetically modified target protein

(cysteine mutation or small peptide tag insertion) example: Tetramethylrhodamine maleimide (TMR)

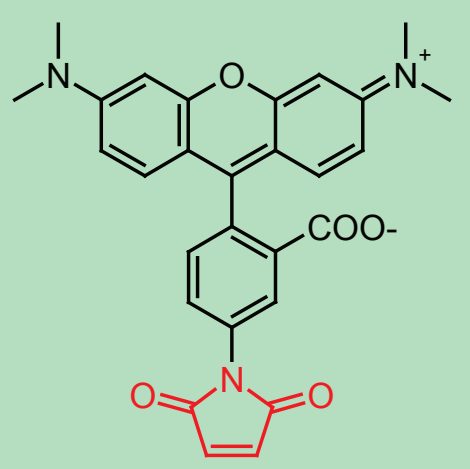

Cysteine-reactive group 


\section{LIGHT-SENSITIVE UAAS}

\section{MOLECULAR}

COMPONENTS

(i) genetically modified ion channel (amber mutation)

(ii) genes for tRNA/synthetase pair

(iii) UAA or UAA-dipeptide of choice

MODE OF INTRODUCTION

EFFICIENCY OF
INCORPORATION

During protein biosynthesis (addition of UAA into the growth medium or cellular injection)

In theory, $100 \%$ (but see below for the 'leakage' issue)

MOLECULAR SIZE

BIOCHEMICAL DIVERSITY

VERSATILITY OF SITE INSERTION

LIGHT PROPERTIES

SUSTAINABILITY

TIME WINDOW IN VIVO

POSSIBLE SIDE- AND OFFTARGET EFFECTS
Small: single photo-reactive side-chain of aromatic nature

Lower: limited by the co-evolution of a bio-orthogonal tRNA/synthetase pair

In principle, 'absolute' (only minor steric restrictions)

Usually in the UV range; red-shifted photo-switching UAAs are under development

Constant turn-over of the tRNA; supply of the UAA may be limiting

\section{Unknown}

- Premature stop at amber codon $\rightarrow$ truncated subunits

- 'Leakage', i.e. unspecific read-through at the introduced amber codon $\rightarrow$ ion channels carrying endogenous amino acids

- Incorporation of UAAs at natural amber codons $\rightarrow$ modifications of the proteome

\section{PHOTO-SENSITIVE TETHERED LIGANDS (PTLS)}

(I) genetically modified ion channel (cysteine mutation)

(ii) PTL of choice

(iii) reducing agents if necessary

Post-translational extra-cellular labeling

$<100 \% \rightarrow$ mixed populations with 'non-labeled' channels

Bulkier multi-component molecule; easily adjustable in length

High: only limited by the feasibility of the PTL chemical synthesis

Only extra-cellular, solvent-accessible regions

Cis-isoform: $360-400 \mathrm{~nm}$ (UV range), trans-isoform: 460-560 nm (blue/green range) or darkness; red-shifted versions available

Re-labeling required

Tens of minutes

- Unspecific cysteine conjugation $\rightarrow$ PTLs at false ion channel positions or within other proteins

- in vivo, off-target effects of PTLs acting as soluble ligands on other proteins 


\section{TRENDS}

- Optopharmacological approaches allow to engineer light-sensitivity into ion channels that are naturally not responsive to optical stimulation.

- Genetically-encoded light-sensitive unnatural amino acids (UAAs) as single sidechain probes provide direct and highly precise optical control over ion channel function.

- Recent fluorescent UAA probes with high environmental sensitivity can accurately track ion channel conformational rearrangements.

- Photo-cleavable, photo-caged, photo-crosslinking and photo-switchable UAAs allow real-time optical control over ion channel structure, gating, pharmacology, and interaction with auxiliary proteins.

- Recent advances of utilizing UAAs in vivo opens up new vistas to study biological ion channel processes in interrelation to their native environment. 